\title{
ANALYSIS OF A COUPLED BEM/FEM EIGENSOLVER FOR THE HYDROELASTIC VIBRATIONS PROBLEM*
}

\author{
Mauricio A. Barrientos ${ }^{1}$, Gabriel N. Gatica ${ }^{2, \dagger}$, Rodolfo Rodríguez ${ }^{2, \dagger}$ And \\ MARCEla E. TORREJón ${ }^{2}$
}

\begin{abstract}
A coupled finite/boundary element method to approximate the free vibration modes of an elastic structure containing an incompressible fluid is analyzed in this paper. The effect of the fluid is taken into account by means of one of the most usual procedures in engineering practice: an added mass formulation, which is posed in terms of boundary integral equations. Piecewise linear continuous elements are used to discretize the solid displacements and the fluid-solid interface variables. Spectral convergence is proved and error estimates are settled for the approximate eigenfunctions and their corresponding vibration frequencies. Implementation issues are also discussed and numerical experiments are reported.
\end{abstract}

Mathematics Subject Classification. 65N25, 65N30, 65N38, 70J30, 74F10, 76Q05.

Received: May 15, 2003. Revised: April 8, 2004.

\section{INTRODUCTION}

In this paper we analyze a coupled boundary/finite element method to numerically solve a spectral problem arising in fluid-solid interactions: the computation of free hydroelastic vibrations. In particular, we consider the problem of determining the harmonic vibrations of a coupled system consisting of an elastic vessel containing an incompressible fluid.

The most direct approach to solve this problem is to discretize a coupled formulation in terms of solid displacements and fluid pressure (see for instance [23]). However, such strategy leads to non symmetric eigenvalue problems, which are usually hard to solve.

Another procedure has been considered in [5] (see also [3] and [4]). It is based on using displacement variables to describe the fluid, discretized by lowest-degree Raviart-Thomas finite elements on a triangular mesh. The interface coupling with classical piecewise linear finite elements for the solid displacements is weakly imposed, yielding a nonconforming method. It has been proved that no spurious modes arise, as typically happens with other discretizations of pure displacement formulations (see [12]), and error estimates have been settled. Although this method works in two or three dimensions, in the first case it can be conveniently implemented

Keywords and phrases. Fluid-structure interaction, hydroelasticity, added mass, BEM/FEM.

* This work was supported by Project P.I. No 96.013.006-1.1, Dirección de Investigación, Universidad de Concepción, Chile.

1 Departamento de Matemáticas, Facultad de Ciencias Básicas, Universidad de Antofagasta, Chile.

${ }^{2}$ GI $^{2}$ MA, Departamento de Ingeniería Matemática, Universidad de Concepción, Casilla 160-C, Concepción, Chile.

e-mail: rodolfo@ing.mat.udec.cl

$\dagger$ Partially supported by FONDAP in Applied Mathematics, Chile. 


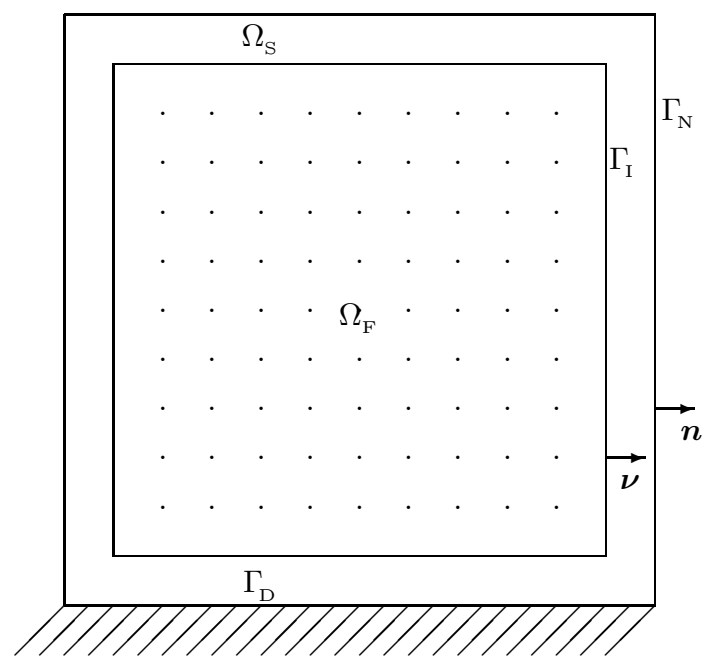

Figure 1. Fluid and solid domains.

by replacing the fluid displacement field by the curl of a stream function. This allows a significant saving of computational effort, since only a scalar magnitude needs to be discretized. However this strategy does not extend to three-dimensional problems.

A third approach consists in eliminating the fluid variables by means of a so-called added mass formulation (see, for instance, [23] or [20]). This is very likely the most used technique in engineering practice to deal with the interaction of an incompressible fluid and an elastic structure. It is based on taking into account the effect of the fluid by means of a Neumann-to-Dirichlet operator (also called the Steklov-Poincaré operator) on the fluid-solid interface. This operator can be further discretized by means of boundary elements or finite elements. For instance, finite element discretizations of this problem have been recently analyzed in [6] and [21].

On the other hand, there are many publications on fluid-solid interaction problems which make use of coupling procedures between boundary and finite element methods. However, most of them are of engineering nature without rigorous justifications. To the author's best knowledge, the first paper in this direction is [16], although for the case of a compressible fluid. More recently, a complete analysis of the fluid-solid problem from both engineering and mathematical point of views has been given in [15] (see also [13]).

In the present paper we propose and analyze a coupled boundary/finite element method for the numerical solution of the added mass formulation described above. We perform this analysis in the two-dimensional case, but the method can be readily extended to three-dimensional problems. In both cases it leads to convenient symmetric eigenvalue problems and does not involve vector variables for the fluid.

The outline of the paper is as follows. In Section 2, we introduce some equivalent variational formulations of the spectral problem, involving integral equations to deal with the Neumann-to-Dirichlet operator. We recall several properties of the basic boundary integral operators which will be used in the sequel. We end this section by introducing a functional settling to analyze the spectral problem which allows us to characterize its solutions and to state their regularity properties. The discrete problem is introduced and analyzed in Section 3, where error estimates are proved both for eigenfunctions and eigenvalues. Some implementation topics are discussed in Section 4, including an equivalent formulation of the discrete spectral problem which turns out to be more convenient from the computational point of view. Finally, we report in this section some numerical experiments.

\section{THE MODEL PROBLEM}

We consider the problem of determining the vibration modes of a linear elastic structure in contact with an incompressible, inviscid and barotropic fluid. Our model problem consists of a two-dimensional vessel completely filled with fluid, as shown in Figure 1. 
Let $\Omega_{\mathrm{F}}$ and $\Omega_{\mathrm{S}}$ be polygonal domains in $\mathbb{R}^{2}$ occupied by the fluid and the solid, respectively. Let us denote by $\Gamma_{\mathrm{I}}$ the interface between solid and fluid and by $\boldsymbol{\nu}$ its unit normal vector pointing outwards $\Omega_{\mathrm{F}}$. The external solid boundary is assumed to be the union of two parts: $\Gamma_{\mathrm{D}}$ and $\Gamma_{\mathrm{N}}$; the structure is supposed to be free along $\Gamma_{\mathrm{N}}$ and fixed along $\Gamma_{\mathrm{D}}$ (for simplicity, meas $\left(\Gamma_{\mathrm{D}}\right)>0$ is also assumed). Finally, $\boldsymbol{n}$ denotes the unit outward normal vector along $\Gamma_{\mathrm{N}}$.

Throughout this paper we will use standard notation for Sobolev spaces and norms. Furthermore, we will denote by $C$ a generic constant not necessarily the same at each occurrence.

The physical magnitudes of the fluid will be denoted by:

- $\boldsymbol{w}$ : the displacement vector field;

- $p$ : the pressure;

- $\rho_{\mathrm{F}}$ : the density;

and those of the solid by:

- $\boldsymbol{u}$ : the displacement vector field;

- $\rho_{\mathrm{S}}$ : the density;

- $\lambda_{\mathrm{S}}$ and $\mu_{\mathrm{S}}$ : the Lamé coefficients, which are defined by

$$
\lambda_{\mathrm{S}}:=\frac{\nu_{\mathrm{S}} E_{\mathrm{S}}}{\left(1-2 \nu_{\mathrm{S}}\right)\left(1+\nu_{\mathrm{S}}\right)} \quad \text { and } \quad \mu_{\mathrm{S}}:=\frac{E_{\mathrm{S}}}{2\left(1+\nu_{\mathrm{S}}\right)}
$$

where $E_{\mathrm{S}}>0$ is the Young modulus and $\nu_{\mathrm{S}} \in\left(0, \frac{1}{2}\right)$ is the Poisson ratio;

- $\varepsilon(\boldsymbol{u})$ : the strain tensor defined by $\varepsilon_{i j}(\boldsymbol{u}):=\frac{1}{2}\left(\frac{\partial u_{i}}{\partial x_{j}}+\frac{\partial u_{j}}{\partial x_{i}}\right), i, j=1,2$;

- $\boldsymbol{\sigma}(\boldsymbol{u})$ : the stress tensor, which we assume related to the strains by Hooke's law:

$$
\sigma_{i j}(\boldsymbol{u})=\lambda_{\mathrm{S}} \sum_{k=1}^{2} \varepsilon_{k k}(\boldsymbol{u}) \delta_{i j}+2 \mu_{\mathrm{s}} \varepsilon_{i j}(\boldsymbol{u}), \quad i, j=1,2 .
$$

In absence of external forces, the classical linearizing procedure yields the following equations for the free small amplitude motions of the fluid-solid system:

$$
\begin{array}{rll}
\operatorname{div}[\boldsymbol{\sigma}(\boldsymbol{u})]=\rho_{\mathrm{S}} \ddot{\boldsymbol{u}} & \text { in } \Omega_{\mathrm{S}}, & t>0, \\
\boldsymbol{\sigma}(\boldsymbol{u}) \boldsymbol{n}=\mathbf{0} & \text { on } \Gamma_{\mathrm{N}}, & t \geq 0 \\
\boldsymbol{u}=\mathbf{0} & \text { on } \Gamma_{\mathrm{D}}, & t \geq 0 \\
\boldsymbol{\sigma}(\boldsymbol{u}) \boldsymbol{\nu}+p \boldsymbol{\nu}=\mathbf{0} & \text { on } \Gamma_{\mathrm{I}}, & t \geq 0 \\
\boldsymbol{w} \cdot \boldsymbol{\nu}=\boldsymbol{u} \cdot \boldsymbol{\nu} & \text { on } \Gamma_{\mathrm{I}}, & t \geq 0 \\
-\boldsymbol{\nabla} p=\rho_{\mathrm{F}} \ddot{\boldsymbol{w}} & \text { in } \Omega_{\mathrm{F}}, & t>0 \\
\operatorname{div} \dot{\boldsymbol{w}}=0 & \text { in } \Omega_{\mathrm{F}}, & t \geq 0
\end{array}
$$

where the dots denote time-derivatives.

The natural vibration modes of the coupled system are given by the harmonic in time solutions of these equations:

$$
\begin{aligned}
& \boldsymbol{u}(x, t)=\boldsymbol{u}(x) \cos \omega t, \quad x \in \Omega_{\mathrm{S}}, \quad t \geq 0, \\
& p(x, t)=p(x) \cos \omega t, \quad x \in \Omega_{\mathrm{F}}, \quad t \geq 0, \\
& \boldsymbol{w}(x, t)=\boldsymbol{w}(x) \cos \omega t, \quad x \in \Omega_{\mathrm{F}}, \quad t \geq 0,
\end{aligned}
$$

where $\omega>0$ is the vibration frequency and $\boldsymbol{u}(x), p(x)$, and $\boldsymbol{w}(x)$ are the amplitudes of the corresponding magnitudes (which throughout the paper will be denoted as the magnitudes themselves). 
For any solution of this form, $\nabla p=\omega^{2} \rho_{\mathrm{F}} \boldsymbol{w}$ and, hence, there exists a fluid displacement potential given by

$$
\varphi:=\frac{p}{\omega^{2} \rho_{\mathrm{F}}},
$$

such that $\boldsymbol{w}=\nabla \varphi$.

Both, pressure and displacement fields in the fluid can then be written in terms of this potential, what leads to the following spectral problem for the free vibration modes of the fluid-structure system:

Find $\omega>0, \boldsymbol{u} \in \mathrm{H}^{1}\left(\Omega_{\mathrm{S}}\right)^{2}$, and $\varphi \in \mathrm{H}^{1}\left(\Omega_{\mathrm{F}}\right),(\boldsymbol{u}, \varphi) \neq \mathbf{0}$, such that:

$$
\begin{array}{rc}
-\operatorname{div}[\boldsymbol{\sigma}(\boldsymbol{u})]=\omega^{2} \rho_{\mathrm{S}} \boldsymbol{u} & \text { in } \Omega_{\mathrm{S}}, \\
\boldsymbol{\sigma}(\boldsymbol{u}) \boldsymbol{n}=\mathbf{0} & \text { on } \Gamma_{\mathrm{N}}, \\
\boldsymbol{u}=\mathbf{0} & \text { on } \Gamma_{\mathrm{D}}, \\
-\boldsymbol{\sigma}(\boldsymbol{u}) \boldsymbol{\nu}=\omega^{2} \rho_{\mathrm{F}} \varphi \boldsymbol{\nu} & \text { on } \Gamma_{\mathrm{I}}, \\
\frac{\partial \varphi}{\partial \nu}=\boldsymbol{u} \cdot \boldsymbol{\nu} & \text { on } \Gamma_{\mathrm{I}}, \\
\Delta \varphi=0 & \text { in } \Omega_{\mathrm{F}} .
\end{array}
$$

To obtain a variational formulation of this problem, we test equation (2.1) with vector fields in the space $\mathrm{H}_{\Gamma_{\mathrm{D}}}^{1}\left(\Omega_{\mathrm{S}}\right)^{2}:=\left\{\boldsymbol{v} \in \mathrm{H}^{1}\left(\Omega_{\mathrm{S}}\right)^{2}:\left.\boldsymbol{v}\right|_{\Gamma_{\mathrm{D}}}=\mathbf{0}\right\}$. Thus, integrating by parts and using (2.2) and (2.4) we have

$$
\int_{\Omega_{\mathrm{S}}} \boldsymbol{\sigma}(\boldsymbol{u}): \boldsymbol{\varepsilon}(\boldsymbol{v}) \mathrm{d} x=\omega^{2}\left(\int_{\Omega_{\mathrm{S}}} \rho_{\mathrm{S}} \boldsymbol{u} \cdot \boldsymbol{v} \mathrm{d} x+\int_{\Gamma_{\mathrm{I}}} \rho_{\mathrm{F}} \varphi \boldsymbol{v} \cdot \boldsymbol{\nu} \mathrm{d} s\right) \quad \forall \boldsymbol{v} \in \mathrm{H}_{\Gamma_{\mathrm{D}}}^{1}\left(\Omega_{\mathrm{S}}\right)^{2}
$$

The harmonic potential $\varphi$ is in its turn a solution of the Neumann problem (2.6)-(2.5); thus it is determined, up to an additive constant, by the normal component of the solid displacement $\boldsymbol{u} \cdot \boldsymbol{\nu}$. In what follows we use standard boundary integral equations to relate both magnitudes.

To this end, let $E(x, y):=\frac{1}{2 \pi} \log \frac{1}{|x-y|}$ be the fundamental solution of the Laplace operator in $\mathbb{R}^{2}$, and recall Green's representation formula for an interior problem in $\Omega_{\mathrm{F}}$ (see e.g. $[10,17]$ ):

$$
\varphi(x)=\int_{\Gamma_{\mathrm{I}}}\left[E(x, y) \frac{\partial \varphi}{\partial \nu}(y)-\frac{\partial E(x, y)}{\partial \nu(y)} \varphi(y)\right] \mathrm{d} s_{y} \quad \forall x \in \Omega_{\mathrm{F}},
$$

where $\boldsymbol{\nu}(y)$ denotes the unit outward normal to $y \in \Gamma_{\mathrm{I}}$.

Then, using the well known jump conditions of the layer potentials, and according to the transmission condition $(2.5)$, we obtain the following integral equations (see e.g. $[8,10,17]$ ):

$$
\begin{array}{cc}
\varphi=V(\boldsymbol{u} \cdot \boldsymbol{\nu})+\left(\frac{1}{2} I-K\right) \varphi & \text { on } \Gamma_{\mathrm{I}}, \\
W \varphi=\left(\frac{1}{2} I-K^{\prime}\right)(\boldsymbol{u} \cdot \boldsymbol{\nu}) & \text { on } \Gamma_{\mathrm{I}},
\end{array}
$$

where $V, K, K^{\prime}$ and $W$ are the so-called boundary integral operators of the single, double, adjoint of the double and hypersingular layer potentials, respectively. These operators are formally defined for a Lipschitz-continuous 
boundary $\Gamma_{\mathrm{I}}$ by:

$$
\begin{aligned}
&(V \varphi)(x):=\int_{\Gamma_{\mathrm{I}}} E(x, y) \varphi(y) \mathrm{d} s_{y}, x \in \Gamma_{\mathrm{I}} \\
&(K \psi)(x):=\int_{\Gamma_{\mathrm{I}}} \frac{\partial E(x, y)}{\partial \nu(y)} \psi(y) \mathrm{d} s_{y}, x \in \Gamma_{\mathrm{I}}, \\
&\left(K^{\prime} \varphi\right)(x):=\int_{\Gamma_{\mathrm{I}}} \frac{\partial E(x, y)}{\partial \nu(x)} \varphi(y) \mathrm{d} s_{y}, \quad x \in \Gamma_{\mathrm{I}} \\
&(W \psi)(x):=-\frac{\partial}{\partial \nu(x)} \int_{\Gamma_{\mathrm{I}}} \frac{\partial E(x, y)}{\partial \nu(y)} \psi(y) \mathrm{d} s_{y}, \quad x \in \Gamma_{\mathrm{I}}
\end{aligned}
$$

Their main mapping properties are collected in the following lemma, where, hereafter, $\langle\cdot, \cdot\rangle$ denotes the duality pairing between $\mathrm{H}^{1 / 2}\left(\Gamma_{\mathrm{I}}\right)$ and $\mathrm{H}^{-1 / 2}\left(\Gamma_{\mathrm{I}}\right)$ with respect to the $\mathrm{L}^{2}\left(\Gamma_{\mathrm{I}}\right)$ inner product,

$$
\mathrm{H}_{0}^{-1 / 2}\left(\Gamma_{\mathrm{I}}\right):=\left\{\varphi \in \mathrm{H}^{-1 / 2}\left(\Gamma_{\mathrm{I}}\right):\langle 1, \varphi\rangle=0\right\} \quad \text { and } \quad \mathrm{H}_{0}^{1 / 2}\left(\Gamma_{\mathrm{I}}\right):=\left\{\psi \in \mathrm{H}^{1 / 2}\left(\Gamma_{\mathrm{I}}\right):\langle\psi, 1\rangle=0\right\} .
$$

Lemma 2.1. The following linear operators are continuous:

$$
\begin{aligned}
& V: \mathrm{H}^{-1 / 2}\left(\Gamma_{\mathrm{I}}\right) \longrightarrow \mathrm{H}^{1 / 2}\left(\Gamma_{\mathrm{I}}\right), \\
& K: \mathrm{H}^{1 / 2}\left(\Gamma_{\mathrm{I}}\right) \longrightarrow \mathrm{H}^{1 / 2}\left(\Gamma_{\mathrm{I}}\right), \\
& K^{\prime}: \mathrm{H}^{-1 / 2}\left(\Gamma_{\mathrm{I}}\right) \longrightarrow \mathrm{H}^{-1 / 2}\left(\Gamma_{\mathrm{I}}\right), \\
& W: \mathrm{H}^{1 / 2}\left(\Gamma_{\mathrm{I}}\right) \longrightarrow \mathrm{H}^{-1 / 2}\left(\Gamma_{\mathrm{I}}\right) .
\end{aligned}
$$

In addition, there holds

$$
K(1)=-\frac{1}{2} \quad \text { and } \quad \operatorname{ker} W=\langle\{1\}\rangle,
$$

and the operators $K$ and $K^{\prime}$ are adjoint with respect to the duality pairing $\langle\cdot, \cdot\rangle$; i.e.,

$$
\langle K \varphi, \psi\rangle=\left\langle\varphi, K^{\prime} \psi\right\rangle \quad \forall \varphi \in \mathrm{H}^{1 / 2}\left(\Gamma_{\mathrm{I}}\right) \quad \forall \psi \in \mathrm{H}^{-1 / 2}\left(\Gamma_{\mathrm{I}}\right) .
$$

Furthermore, $\langle V \cdot, \cdot\rangle$ and $\langle\cdot, W \cdot\rangle$ are symmetric continuous bilinear forms on $\mathrm{H}^{-1 / 2}\left(\Gamma_{\mathrm{I}}\right) \times \mathrm{H}^{-1 / 2}\left(\Gamma_{\mathrm{I}}\right)$ and $\mathrm{H}^{1 / 2}\left(\Gamma_{\mathrm{I}}\right) \times$ $\mathrm{H}^{1 / 2}\left(\Gamma_{\mathrm{I}}\right)$, respectively, and there exist strictly positive constants $\alpha_{0}$ and $\alpha_{1}$ such that

$$
\langle V \varphi, \varphi\rangle \geq \alpha_{0}\|\varphi\|_{\mathrm{H}^{-1 / 2}\left(\Gamma_{\mathrm{I}}\right)}^{2} \quad \forall \varphi \in \mathrm{H}_{0}^{-1 / 2}\left(\Gamma_{\mathrm{I}}\right)
$$

and

$$
\langle\psi, W \psi\rangle \geq \alpha_{1}\|\psi\|_{\mathrm{H}^{1 / 2}\left(\Gamma_{\mathrm{I}}\right)}^{2} \quad \forall \psi \in \mathrm{H}_{0}^{1 / 2}\left(\Gamma_{\mathrm{I}}\right)
$$

Proof. See [8] and [14].

As a consequence of this lemma we also have the following result:

Lemma 2.2. There holds:

(1) $K^{\prime}\left(\mathrm{H}_{0}^{-1 / 2}\left(\Gamma_{\mathrm{I}}\right)\right) \subset \mathrm{H}_{0}^{-1 / 2}\left(\Gamma_{\mathrm{I}}\right)$;

(2) $W$ induces an isomorphism, still denoted by $W$, from $\mathrm{H}_{0}^{1 / 2}\left(\Gamma_{\mathrm{I}}\right)$ onto $\mathrm{H}_{0}^{-1 / 2}\left(\Gamma_{\mathrm{I}}\right)$.

Proof. The first assertion is an immediate consequence of the fact that $K(1)=-\frac{1}{2}$. On the other hand, $W(1)=$ 0 implies $W\left(\mathrm{H}_{0}^{1 / 2}\left(\Gamma_{\mathrm{I}}\right)\right) \subset \mathrm{H}_{0}^{-1 / 2}\left(\Gamma_{\mathrm{I}}\right)$. Thus the second assertion follows from (2.14) and the Lax-Milgram lemma. 
Replacing (2.9) into (2.7) and using (2.10) and (2.12), we obtain that any solution of Problem (2.1)-(2.6) also solves the following variational problem:

Find $\omega \geq 0$ and $\mathbf{0} \neq(\boldsymbol{u}, \varphi) \in \mathrm{H}_{\Gamma_{\mathrm{D}}}^{1}\left(\Omega_{\mathrm{S}}\right)^{2} \times \mathrm{H}^{1 / 2}\left(\Gamma_{\mathrm{I}}\right)$ such that

$$
\int_{\Omega_{\mathrm{S}}} \boldsymbol{\sigma}(\boldsymbol{u}): \boldsymbol{\varepsilon}(\boldsymbol{v}) \mathrm{d} x=\omega^{2}\left[\int_{\Omega_{\mathrm{S}}} \rho_{\mathrm{S}} \boldsymbol{u} \cdot \boldsymbol{v} \mathrm{d} x+\rho_{\mathrm{F}}\langle V(\boldsymbol{u} \cdot \boldsymbol{\nu}), \boldsymbol{v} \cdot \boldsymbol{\nu}\rangle+\rho_{\mathrm{F}}\left\langle\left(\frac{1}{2} I-K\right) \varphi, \boldsymbol{v} \cdot \boldsymbol{\nu}\right\rangle\right] \quad \forall \boldsymbol{v} \in \mathrm{H}_{\Gamma_{\mathrm{D}}}^{1}\left(\Omega_{\mathrm{S}}\right)^{2}
$$

and

$$
\langle\psi, W \varphi\rangle=\left\langle\left(\frac{1}{2} I-K\right) \psi, \boldsymbol{u} \cdot \boldsymbol{\nu}\right\rangle \quad \forall \psi \in \mathrm{H}^{1 / 2}\left(\Gamma_{\mathrm{I}}\right) .
$$

The following lemma shows that this variational problem is actually equivalent to the spectral Problem (2.1)-(2.6):

Lemma 2.3. Problems (2.1)-(2.6) and (2.15)-(2.16) are equivalent. More precisely, there hold:

(1) Let $(\omega, \boldsymbol{u}, \varphi) \in \mathbb{R}^{+} \times \mathrm{H}^{1}\left(\Omega_{\mathrm{S}}\right)^{2} \times \mathrm{H}^{1}\left(\Omega_{\mathrm{F}}\right)$ be a solution of (2.1)-(2.6). Then, $\left(\omega, \boldsymbol{u},\left.\varphi\right|_{\Gamma_{\mathrm{I}}}\right)$ is a solution of (2.15)-(2.16)

(2) Let $(\omega, \boldsymbol{u}, \tilde{\varphi}) \in \mathbb{R}^{+} \times \mathrm{H}_{\Gamma_{\mathrm{D}}}^{1}\left(\Omega_{\mathrm{S}}\right)^{2} \times \mathrm{H}^{1 / 2}\left(\Gamma_{\mathrm{I}}\right)$ be a solution of (2.15)-(2.16). Then, $\exists \varphi \in \mathrm{H}^{1}\left(\Omega_{\mathrm{F}}\right)$ such that $\left.\varphi\right|_{\Gamma_{\mathrm{I}}}=\tilde{\varphi}$ and $(\omega, \boldsymbol{u}, \varphi)$ is a solution of (2.1)-(2.6).

Proof. The first assertion has been already proved above. In order to prove the second one, let $(\omega, \boldsymbol{u}, \tilde{\varphi}) \in$ $\mathbb{R}^{+} \times \mathrm{H}_{\Gamma_{\mathrm{D}}}^{1}\left(\Omega_{\mathrm{S}}\right)^{2} \times \mathrm{H}^{1 / 2}\left(\Gamma_{\mathrm{I}}\right)$ be a solution of (2.15)-(2.16). By testing (2.16) with $\psi=1$ and using (2.11), we have that $\langle 1, \boldsymbol{u} \cdot \boldsymbol{\nu}\rangle=0$. Then, the following Neumann problem is compatible:

$$
\begin{aligned}
\Delta \varphi=0 & \text { in } \Omega_{\mathrm{F}}, \\
\frac{\partial \varphi}{\partial \nu}=\boldsymbol{u} \cdot \boldsymbol{\nu} & \text { on } \Gamma_{\mathrm{I}} .
\end{aligned}
$$

Hence, its solution $\varphi$ is well defined up to an additive constant. Moreover, Green's representation formula (2.8) implies that equations $(2.9)$ and $(2.10)$ hold true (see e.g. $[8,10])$. Then, from $(2.10)$ and $(2.16)$ we have that $W\left(\left.\varphi\right|_{\Gamma_{\mathrm{I}}}\right)=W \tilde{\varphi}$, which according to $(2.11)$ yields that $\left.\varphi\right|_{\Gamma_{\mathrm{I}}}-\tilde{\varphi}$ is constant. Hence, we can choose the additive constant defining uniquely $\varphi \in \mathrm{H}^{1}\left(\Omega_{\mathrm{F}}\right)$ such that $\left.\varphi\right|_{\Gamma_{\mathrm{I}}}=\tilde{\varphi}$.

Now, $\varphi$ clearly satisfies (2.5)-(2.6) and, because of (2.15) and (2.9),

$$
\int_{\Omega_{\mathrm{S}}} \boldsymbol{\sigma}(\boldsymbol{u}): \boldsymbol{\varepsilon}(\boldsymbol{v}) \mathrm{d} x=\omega^{2}\left(\int_{\Omega_{\mathrm{S}}} \rho_{\mathrm{S}} \boldsymbol{u} \cdot \boldsymbol{v} \mathrm{d} x+\int_{\Gamma_{\mathrm{I}}} \rho_{\mathrm{F}} \varphi \boldsymbol{v} \cdot \boldsymbol{\nu} \mathrm{d} x\right) \quad \forall \boldsymbol{v} \in \mathrm{H}_{\Gamma_{\mathrm{D}}}^{1}\left(\Omega_{\mathrm{S}}\right)^{2} .
$$

Finally, (2.1) is obtained by testing this equation with $\boldsymbol{v} \in \mathcal{D}\left(\Omega_{\mathrm{S}}\right)^{2}$, and (2.2) and (2.4) by doing it with arbitrary $\boldsymbol{v} \in \mathrm{H}_{\Gamma_{\mathrm{D}}}^{1}\left(\Omega_{\mathrm{S}}\right)^{2}$. Therefore, we have proved that $(\omega, \boldsymbol{u}, \varphi)$ is a solution of $(2.1)-(2.6)$.

For the theoretical analysis, it will be useful to consider a variational formulation slightly different to $(2.15)-(2.16)$. To this goal, let

$$
\mathcal{V}:=\left\{\boldsymbol{v} \in \mathrm{H}_{\Gamma_{\mathrm{D}}}^{1}\left(\Omega_{\mathrm{S}}\right)^{2}: \int_{\Gamma_{\mathrm{I}}} \boldsymbol{v} \cdot \boldsymbol{\nu} \mathrm{d} s=0\right\}
$$

be the space of admissible solid displacements, in the sense of preserving the volume of $\Omega_{\mathrm{F}}$, and

$$
\mathcal{Q}:=\mathrm{H}_{0}^{1 / 2}\left(\Gamma_{\mathrm{I}}\right) .
$$


We define the following problem:

Find $\omega \geq 0$ and $\mathbf{0} \neq(\boldsymbol{u}, \varphi) \in \mathcal{V} \times \mathcal{Q}$ such that

$$
\int_{\Omega_{\mathrm{S}}} \boldsymbol{\sigma}(\boldsymbol{u}): \boldsymbol{\varepsilon}(\boldsymbol{v}) \mathrm{d} x=\omega^{2}\left[\int_{\Omega_{\mathrm{S}}} \rho_{\mathrm{S}} \boldsymbol{u} \cdot \boldsymbol{v} \mathrm{d} x+\rho_{\mathrm{F}}\langle V(\boldsymbol{u} \cdot \boldsymbol{\nu}), \boldsymbol{v} \cdot \boldsymbol{\nu}\rangle+\rho_{\mathrm{F}}\left\langle\left(\frac{1}{2} I-K\right) \varphi, \boldsymbol{v} \cdot \boldsymbol{\nu}\right\rangle\right] \forall \boldsymbol{v} \in \mathcal{V}
$$

and

$$
\langle\psi, W \varphi\rangle=\left\langle\left(\frac{1}{2} I-K\right) \psi, \boldsymbol{u} \cdot \boldsymbol{\nu}\right\rangle \quad \forall \psi \in \mathcal{Q} .
$$

We show below that this variational formulation is equivalent to (2.15)-(2.16). To this aim, let $\boldsymbol{v}^{0}$ be an arbitrary, but fixed, element in $\mathrm{H}_{\Gamma_{\mathrm{D}}}^{1}\left(\Omega_{\mathrm{S}}\right)^{2}$ such that $\int_{\Gamma_{\mathrm{I}}} \boldsymbol{v}^{0} \cdot \boldsymbol{\nu} \mathrm{d} s \neq 0$. Also, for simplicity of presentation, we denote

$$
B((\boldsymbol{w}, \psi), \boldsymbol{v}):=\int_{\Omega_{\mathrm{S}}} \rho_{\mathrm{S}} \boldsymbol{w} \cdot \boldsymbol{v} \mathrm{d} x+\rho_{\mathrm{F}}\langle V(\boldsymbol{w} \cdot \boldsymbol{\nu}), \boldsymbol{v} \cdot \boldsymbol{\nu}\rangle+\rho_{\mathrm{F}}\left\langle\left(\frac{1}{2} I-K\right) \psi, \boldsymbol{v} \cdot \boldsymbol{\nu}\right\rangle
$$

$\forall \boldsymbol{w}, \boldsymbol{v} \in \mathrm{H}_{\Gamma_{\mathrm{D}}}^{1}\left(\Omega_{\mathrm{S}}\right)^{2}$ and $\forall \psi \in \mathrm{H}^{1 / 2}\left(\Gamma_{\mathrm{I}}\right)$

Lemma 2.4. Problems (2.15)-(2.16) and (2.17)-(2.18) are equivalent. More precisely, there hold:

(1) Let $(\omega, \boldsymbol{u}, \varphi) \in \mathbb{R}^{+} \times \mathrm{H}_{\Gamma_{\mathrm{D}}}^{1}\left(\Omega_{\mathrm{S}}\right)^{2} \times \mathrm{H}^{1 / 2}\left(\Gamma_{\mathrm{I}}\right)$ be a solution of (2.15)-(2.16) and let

$$
\tilde{\varphi}:=\varphi-\frac{1}{\operatorname{meas}\left(\Gamma_{\mathrm{I}}\right)} \int_{\Gamma_{\mathrm{I}}} \varphi \mathrm{d} s
$$

It follows that $(\boldsymbol{u}, \tilde{\varphi}) \in \mathcal{V} \times \mathcal{Q}$ and $(\omega, \boldsymbol{u}, \tilde{\varphi})$ is a solution of (2.17)-(2.18).

(2) Let $(\omega, \boldsymbol{u}, \tilde{\varphi}) \in \mathbb{R}^{+} \times \mathcal{V} \times \mathcal{Q}$ be a solution of (2.17)-(2.18). It follows that $\omega>0$ and $\left(\omega, \boldsymbol{u}, \tilde{\varphi}+c_{0}\right)$ is a solution of (2.15)-(2.16), where

$$
c_{0}:=\frac{\int_{\Omega_{\mathrm{S}}} \boldsymbol{\sigma}(\boldsymbol{u}): \boldsymbol{\varepsilon}\left(\boldsymbol{v}^{0}\right) \mathrm{d} x-\omega^{2} B\left((\boldsymbol{u}, \tilde{\varphi}), \boldsymbol{v}^{0}\right)}{\omega^{2} \rho_{\mathrm{F}} \int_{\Gamma_{\mathrm{I}}} \boldsymbol{v}^{0} \cdot \boldsymbol{\nu} \mathrm{d} s} .
$$

Proof. Let $(\omega, \boldsymbol{u}, \varphi) \in \mathbb{R}^{+} \times \mathrm{H}_{\Gamma_{\mathrm{D}}}^{1}\left(\Omega_{\mathrm{S}}\right)^{2} \times \mathrm{H}^{1 / 2}\left(\Gamma_{\mathrm{I}}\right)$ be a solution of (2.15)-(2.16) and $\tilde{\varphi}$ as defined above. It is clear that $\tilde{\varphi} \in \mathcal{Q}$. Also taking $\psi=1$ in (2.16) and using (2.11) we have that $\boldsymbol{u} \in \mathcal{V}$. Next, it is easy to see that $\left\langle\left(\frac{1}{2} I-K\right) \varphi, \boldsymbol{v} \cdot \boldsymbol{\nu}\right\rangle=\left\langle\left(\frac{1}{2} I-K\right) \tilde{\varphi}, \boldsymbol{v} \cdot \boldsymbol{\nu}\right\rangle \forall \boldsymbol{v} \in \mathcal{V}$ and hence $(2.15)$ gives

$$
\int_{\Omega_{\mathrm{S}}} \boldsymbol{\sigma}(\boldsymbol{u}): \boldsymbol{\varepsilon}(\boldsymbol{v}) \mathrm{d} x=\omega^{2} B((\boldsymbol{u}, \tilde{\varphi}), \boldsymbol{v}) \quad \forall \boldsymbol{v} \in \mathcal{V} .
$$

In addition, since $W(1)=0$ we obtain $W \varphi=W \tilde{\varphi}$, and therefore (2.16) yields

$$
\langle\psi, W \tilde{\varphi}\rangle=\left\langle\left(\frac{1}{2} I-K\right) \psi, \boldsymbol{u} \cdot \boldsymbol{\nu}\right\rangle \quad \forall \psi \in \mathcal{Q} .
$$

Thus we have proved that $(\omega, \boldsymbol{u}, \varphi)$ is a solution of $(2.17)-(2.18)$.

Conversely, let $(\omega, \boldsymbol{u}, \tilde{\varphi}) \in \mathbb{R}^{+} \times \mathcal{V} \times \mathcal{Q}$ be a solution of (2.17)-(2.18). We first observe that $\boldsymbol{u} \neq 0$ since otherwise (2.18) and Lemma 2.2(2) would imply that $\tilde{\varphi}$ would also vanish. Then, from (2.17) and Korn's inequality we deduce that $\omega>0$. 
Next, let $\varphi=\tilde{\varphi}+c_{0}$, with $c_{0}$ as defined above. From the definition of $c_{0}$ we have that

$$
\int_{\Omega_{\mathrm{S}}} \boldsymbol{\sigma}(\boldsymbol{u}): \boldsymbol{\varepsilon}\left(\boldsymbol{v}^{0}\right) \mathrm{d} x=\omega^{2} B\left((\boldsymbol{u}, \tilde{\varphi}), \boldsymbol{v}^{0}\right)+\omega^{2} \rho_{\mathrm{F}} \int c_{0} \boldsymbol{v}^{0} \cdot \boldsymbol{\nu} \mathrm{d} s=\omega^{2} B\left((\boldsymbol{u}, \varphi), \boldsymbol{v}^{0}\right),
$$

whereas, since $\left\langle\left(\frac{1}{2} I-K\right) \varphi, \boldsymbol{v} \cdot \boldsymbol{\nu}\right\rangle=\left\langle\left(\frac{1}{2} I-K\right) \tilde{\varphi}, \boldsymbol{v} \cdot \boldsymbol{\nu}\right\rangle \forall \boldsymbol{v} \in \mathcal{V}$, we have from (2.17) that

$$
\int_{\Omega_{\mathrm{S}}} \boldsymbol{\sigma}(\boldsymbol{u}): \varepsilon(\boldsymbol{v}) \mathrm{d} x=\omega^{2} B((\boldsymbol{u}, \tilde{\varphi}), \boldsymbol{v})=\omega^{2} B((\boldsymbol{u}, \varphi), \boldsymbol{v}) \quad \forall \boldsymbol{v} \in \mathcal{V} .
$$

Then, since $\mathrm{H}_{\Gamma_{\mathrm{D}}}^{1}\left(\Omega_{\mathrm{S}}\right)^{2}=\mathcal{V} \oplus\left\langle\left\{\boldsymbol{v}^{0}\right\}\right\rangle$, we conclude that $(\omega, \boldsymbol{u}, \varphi)$ satisfies (2.15).

On the other hand, given $\psi \in \mathrm{H}^{1 / 2}\left(\Gamma_{\mathrm{I}}\right)$ let

$$
\tilde{\psi}:=\psi-\frac{1}{\operatorname{meas}\left(\Gamma_{\mathrm{I}}\right)} \int_{\Gamma_{\mathrm{I}}} \psi \mathrm{d} s .
$$

Then $\tilde{\psi} \in \mathcal{Q}$ and, hence, using (2.18) and the fact that $W(1)=0$, we obtain

$$
\left\langle\psi, W\left(\tilde{\varphi}+c_{0}\right)\right\rangle=\langle\tilde{\psi}, W \tilde{\varphi}\rangle=\left\langle\left(\frac{1}{2} I-K\right) \tilde{\psi}, \boldsymbol{u} \cdot \boldsymbol{\nu}\right\rangle=\left\langle\left(\frac{1}{2} I-K\right) \psi, \boldsymbol{u} \cdot \boldsymbol{\nu}\right\rangle .
$$

Thus $(\omega, \boldsymbol{u}, \varphi)$ satisfies $(2.16)$ and we conclude the proof.

A last convenient form of this problem can be obtained by eliminating $\varphi \in \mathcal{Q}$. In fact, from (2.18) and Lemma 2.2, we have that

$$
\varphi=W^{-1}\left(\frac{1}{2} I-K^{\prime}\right)(\boldsymbol{u} \cdot \boldsymbol{\nu})
$$

Then, the right hand side of (2.17) can be written in terms of the operator

$$
M:=V+\left(\frac{1}{2} I-K\right) W^{-1}\left(\frac{1}{2} I-K^{\prime}\right): \mathrm{H}_{0}^{-1 / 2}\left(\Gamma_{\mathrm{I}}\right) \longrightarrow \mathrm{H}^{1 / 2}\left(\Gamma_{\mathrm{I}}\right) / \mathbb{R} .
$$

This is the well known Steklov-Poincaré or NtD (Neumann to Dirichlet) operator which, to a given Neumann data $g \in \mathrm{H}_{0}^{-1 / 2}\left(\Gamma_{\mathrm{I}}\right)$, associates $M g=\left.z\right|_{\Gamma_{\mathrm{I}}} \in \mathrm{H}^{1 / 2}\left(\Gamma_{\mathrm{I}}\right) / \mathbb{R}$, with $z \in \mathrm{H}^{1}\left(\Omega_{\mathrm{F}}\right) / \mathbb{R}$ being the solution of the compatible Neumann problem (see e.g. [17])

$$
\begin{array}{ll}
\Delta z=0 & \text { in } \Omega_{\mathrm{F}}, \\
\frac{\partial z}{\partial \nu}=g & \text { on } \Gamma_{\mathrm{I}} .
\end{array}
$$

Note that $\langle M g, \boldsymbol{v} \cdot \boldsymbol{\nu}\rangle$ is well defined $\forall \boldsymbol{v} \in \mathcal{V}$, because $\langle 1, \boldsymbol{v} \cdot \boldsymbol{\nu}\rangle=0$. Then, any solution of (2.17)-(2.18) also satisfies the following problem:

Find $\omega \geq 0$ and $\mathbf{0} \neq \boldsymbol{u} \in \mathcal{V}$ such that

$$
\int_{\Omega_{\mathrm{S}}} \boldsymbol{\sigma}(\boldsymbol{u}): \boldsymbol{\varepsilon}(\boldsymbol{v}) \mathrm{d} x=\omega^{2}\left[\int_{\Omega_{\mathrm{S}}} \rho_{\mathrm{S}} \boldsymbol{u} \cdot \boldsymbol{v} \mathrm{d} x+\rho_{\mathrm{F}}\langle M(\boldsymbol{u} \cdot \boldsymbol{\nu}), \boldsymbol{v} \cdot \boldsymbol{\nu}\rangle\right] \quad \forall \boldsymbol{v} \in \mathcal{V} .
$$

In the context of fluid-structure interactions $\rho_{\mathrm{F}} M$ is called the added mass operator, since it condenses the whole effect of the incompressible fluid on an integral on the wetted solid boundary (i.e., on the fluid-solid interface).

The following lemma shows that the spectral problem above is actually equivalent to Problem (2.17)-(2.18). 
Lemma 2.5. Problems (2.17)-(2.18) and (2.19) are equivalent. More precisely, there hold:

(1) Let $(\omega, \boldsymbol{u}, \tilde{\varphi}) \in \mathbb{R}^{+} \times \mathcal{V} \times \mathcal{Q}$ be a solution of (2.17)-(2.18). Then $(\omega, \boldsymbol{u})$ is a solution of (2.19).

(2) Let $(\omega, \boldsymbol{u}) \in \mathbb{R}^{+} \times \mathcal{V}$ be a solution of (2.19). Then, there exists $\varphi \in \mathcal{Q}$ such that $(\omega, \boldsymbol{u}, \varphi)$ is a solution of (2.17)-(2.18).

Proof. The first assertion has been already proved above. To prove the second one, let $(\omega, \boldsymbol{u}) \in \mathbb{R}^{+} \times \mathcal{V}$ be a solution of (2.19). Let $\varphi=W^{-1}\left(\frac{1}{2} I-K^{\prime}\right) \boldsymbol{u} \cdot \boldsymbol{\nu} \in \mathrm{H}_{0}^{1 / 2}\left(\Gamma_{\mathrm{I}}\right)$. Then, clearly $(\omega, \boldsymbol{u}, \varphi) \in \mathbb{R}^{+} \times \mathcal{V} \times \mathcal{Q}$ is a solution of $(2.17)-(2.18)$.

Thus, as a consequence of the three previous lemmas we have the following result:

Theorem 2.6. Spectral problems (2.1)-(2.6), (2.15)-(2.16), (2.17)-(2.18) and (2.19) are equivalent.

The solutions of the variational spectral problem (2.19) are known to be a sequence of strictly positive vibration frequencies $\omega_{n} \rightarrow \infty$, each of them with a finite dimensional subspace of functions $\boldsymbol{u} \in \mathcal{V}$ satisfying (2.19) (see for instance [6]). Anyway, we shall obtain this result again, since it is an immediate consequence of a regularizing property of the source problem associated to (2.19), which will be used below to prove spectral convergence results.

Let $a$ and $b$ be the continuous bilinear forms on $\mathcal{V} \times \mathcal{V}$ defined by

$$
\begin{aligned}
a(\boldsymbol{u}, \boldsymbol{v}) & :=\int_{\Omega_{\mathrm{S}}} \boldsymbol{\sigma}(\boldsymbol{u}): \boldsymbol{\varepsilon}(\boldsymbol{v}) \mathrm{d} x \\
b(\boldsymbol{u}, \boldsymbol{v}) & :=\int_{\Omega_{\mathrm{S}}} \rho_{\mathrm{S}} \boldsymbol{u} \cdot \boldsymbol{v} \mathrm{d} x+\rho_{\mathrm{F}}\langle M(\boldsymbol{u} \cdot \boldsymbol{\nu}), \boldsymbol{v} \cdot \boldsymbol{\nu}\rangle
\end{aligned}
$$

The bilinear form $a$ is symmetric and, by Korn's inequality, elliptic. Regarding $b$ we have the following result:

Lemma 2.7. The bilinear form $b$ is symmetric and positive definite; moreover,

$$
b(\boldsymbol{v}, \boldsymbol{v}) \geq \rho_{\mathrm{S}}\|\boldsymbol{v}\|_{\mathrm{L}^{2}\left(\Omega_{\mathrm{S}}\right)^{2}}^{2}+\rho_{\mathrm{F}} \alpha_{0}\|\boldsymbol{v} \cdot \boldsymbol{\nu}\|_{\mathrm{H}^{-1 / 2}\left(\Gamma_{\mathrm{I}}\right)}^{2}>0 \quad \forall \boldsymbol{v} \in \mathcal{V}: \boldsymbol{v} \neq \mathbf{0} .
$$

Proof. The symmetry is consequence of the definition of $M$, the symmetry of $\langle V \cdot, \cdot\rangle$ and $\langle\cdot, W \cdot\rangle$, and Lemma 2.2 . To prove the inequality yielding the positive definiteness, note that from the definition of $b$ we have

$$
b(\boldsymbol{v}, \boldsymbol{v})=\rho_{\mathrm{S}}\|\boldsymbol{v}\|_{\mathrm{L}^{2}\left(\Omega_{\mathrm{S}}\right)^{2}}^{2}+\rho_{\mathrm{F}}\langle V(\boldsymbol{v} \cdot \boldsymbol{\nu}), \boldsymbol{v} \cdot \boldsymbol{\nu}\rangle+\rho_{\mathrm{F}}\left\langle\left(\frac{1}{2} I-K\right) W^{-1}\left(\frac{1}{2} I-K^{\prime}\right)(\boldsymbol{v} \cdot \boldsymbol{\nu}), \boldsymbol{v} \cdot \boldsymbol{\nu}\right\rangle .
$$

The last term in the right-hand side above is non-negative as a consequence of 2.12, Lemma 2.2, and (2.14). Then (2.13) allows us to conclude the proof.

The above lemma shows that $b$ is an inner product on $\mathcal{V}$. Then, it defines a norm on this space that we denote $|\cdot|$, namely,

$$
|\boldsymbol{v}|:=b(\boldsymbol{v}, \boldsymbol{v})^{1 / 2}=\left[\int_{\Omega_{\mathrm{S}}} \rho_{\mathrm{S}}|\boldsymbol{v}|^{2} \mathrm{~d} x+\rho_{\mathrm{F}}\langle M(\boldsymbol{v} \cdot \boldsymbol{\nu}), \boldsymbol{v} \cdot \boldsymbol{\nu}\rangle\right]^{1 / 2} \boldsymbol{v} \in \mathcal{V}
$$

This norm will play a role in the last section for proving a double order of convergence for the approximation of the vibration frequencies.

Let $T: \mathcal{V} \longrightarrow \mathcal{V}$ be the linear bounded operator defined for any $f \in \mathcal{V}$ by:

$$
T \boldsymbol{f}:=\boldsymbol{u} \in \mathcal{V}: \quad a(\boldsymbol{u}, \boldsymbol{v})=b(\boldsymbol{f}, \boldsymbol{v}) \quad \forall \boldsymbol{v} \in \mathcal{V}
$$


Because of the symmetry of $a$ and $b, T$ is self-adjoint with respect to both bilinear forms; thus all of its eigenvalues are real and positive. Furthermore, clearly $(\lambda, \boldsymbol{u})$ is an eigenpair of $T$ if and only if $\omega:=\frac{1}{\sqrt{\lambda}}$ and $\boldsymbol{u}$ are solution of (2.19).

We omit the proof of the following lemma, regarding a regularizing property of $T$, since it is essentially the same as that of Proposition 4.3 in [6]:

Lemma 2.8. There exist constants $t \in(0,1]$ and $C>0$ such that if $\boldsymbol{u}=T \boldsymbol{f}$, with $\boldsymbol{f} \in \mathcal{V}$, then $\boldsymbol{u} \in \mathrm{H}^{1+t}\left(\Omega_{\mathrm{S}}\right)^{2}$ and

$$
\|\boldsymbol{u}\|_{\mathrm{H}^{1+t}\left(\Omega_{\mathrm{S}}\right)^{2}} \leq C|\boldsymbol{f}| .
$$

The constant $t$ in this lemma depends on the reentrant angles of $\Omega_{\mathrm{S}}$, the angles between $\Gamma_{\mathrm{D}}$ and $\Gamma_{\mathrm{N}}$, and the Lamé coefficients (see [11], as well as [18,19] for further results on mixed Laplace-Lamé transmission problems).

As an immediate consequence of Lemma 2.8 and the compact inclusion $\mathrm{H}^{1+t}\left(\Omega_{\mathrm{S}}\right)^{2} \cap \mathcal{V} \hookrightarrow \mathcal{V}, T$ is a compact operator and the following spectral characterization holds:

Theorem 2.9. The spectrum of $T$ consists of $\lambda=0$ and a sequence of strictly positive finite multiplicity eigenvalues $\left\{\lambda_{n}: n \in \mathbb{N}\right\}$.

\section{DisCRETE ANALYSIS}

To discretize problem (2.17)-(2.18) we use standard finite elements. Let $\left\{\mathcal{T}_{h}^{\mathrm{S}}\right\}$ be a family of regular triangulations of $\Omega_{\mathrm{S}}$, where $h$ stands for the mesh-size. Let $\left\{\mathcal{T}_{h}^{\mathrm{I}}\right\}$ be the family of meshes induced by $\mathcal{T}_{h}^{\mathrm{S}}$ on $\Gamma_{\mathrm{I}}$. Let $\mathcal{L}_{h}\left(\Omega_{\mathrm{S}}\right)$ and $\mathcal{L}_{h}\left(\Gamma_{\mathrm{I}}\right)$ be the spaces of continuous piecewise linear functions on $\mathcal{T}_{h}^{\mathrm{S}}$ and $\mathcal{T}_{h}^{\mathrm{I}}$, respectively. Consider the following finite dimensional subspaces of $\mathcal{V}$ and $\mathcal{Q}$ :

$$
\begin{aligned}
& \mathcal{V}_{h}:=\left\{\boldsymbol{v}_{h} \in \mathcal{L}_{h}\left(\Omega_{\mathrm{S}}\right)^{2}:\left.\boldsymbol{v}_{h}\right|_{\Gamma_{\mathrm{D}}}=0 \text { and } \int_{\Gamma_{\mathrm{I}}} \boldsymbol{v}_{h} \cdot \boldsymbol{\nu} \mathrm{d} s=0\right\}, \\
& \mathcal{Q}_{h}:=\mathcal{L}_{h}\left(\Gamma_{\mathrm{I}}\right) \cap \mathrm{H}_{0}^{1 / 2}\left(\Gamma_{\mathrm{I}}\right) .
\end{aligned}
$$

Thus, we obtain the following discrete version of problem (2.17)-(2.18):

Find $\omega_{h} \geq 0$ and $\mathbf{0} \neq\left(\boldsymbol{u}_{h}, \varphi_{h}\right) \in \mathcal{V}_{h} \times \mathcal{Q}_{h}$ such that

$$
\int_{\Omega_{\mathrm{S}}} \boldsymbol{\sigma}\left(\boldsymbol{u}_{h}\right): \varepsilon\left(\boldsymbol{v}_{h}\right) \mathrm{d} x=\omega_{h}^{2}\left[\int_{\Omega_{\mathrm{S}}} \rho_{\mathrm{S}} \boldsymbol{u}_{h} \cdot \boldsymbol{v}_{h} \mathrm{~d} x+\rho_{\mathrm{F}}\left\langle V\left(\boldsymbol{u}_{h} \cdot \boldsymbol{\nu}\right), \boldsymbol{v}_{h} \cdot \boldsymbol{\nu}\right\rangle+\rho_{\mathrm{F}}\left\langle\left(\frac{1}{2} I-K\right) \varphi_{h}, \boldsymbol{v}_{h} \cdot \boldsymbol{\nu}\right\rangle\right]
$$

and

$$
\left\langle\psi_{h}, W \varphi_{h}\right\rangle=\left\langle\left(\frac{1}{2} I-K\right) \psi_{h}, \boldsymbol{u}_{h} \cdot \boldsymbol{\nu}\right\rangle \quad \forall \psi_{h} \in \mathcal{Q}_{h}
$$

Let $b_{h}$ be the continuous bilinear form on $\mathcal{V} \times \mathcal{V}$ defined by

$$
b_{h}(\boldsymbol{u}, \boldsymbol{v}):=\int_{\Omega_{\mathrm{S}}} \rho_{\mathrm{S}} \boldsymbol{u} \cdot \boldsymbol{v} \mathrm{d} x+\rho_{\mathrm{F}}\langle V(\boldsymbol{u} \cdot \boldsymbol{\nu}), \boldsymbol{v} \cdot \boldsymbol{\nu}\rangle+\rho_{\mathrm{F}}\left\langle\left(\frac{1}{2} I-K\right) \varphi_{h}, \boldsymbol{v} \cdot \boldsymbol{\nu}\right\rangle,
$$

with $\varphi_{h} \in \mathcal{Q}_{h}$ being the solution of

$$
\left\langle\psi_{h}, W \varphi_{h}\right\rangle=\left\langle\left(\frac{1}{2} I-K\right) \psi_{h}, \boldsymbol{u} \cdot \boldsymbol{\nu}\right\rangle \quad \forall \psi_{h} \in \mathcal{Q}_{h} .
$$


This bilinear form is also symmetric. Indeed, for $\boldsymbol{u}, \boldsymbol{v} \in \mathcal{V}$,

$$
b_{h}(\boldsymbol{v}, \boldsymbol{u})=\int_{\Omega_{\mathrm{S}}} \rho_{\mathrm{S}} \boldsymbol{v} \cdot \boldsymbol{u} \mathrm{d} x+\rho_{\mathrm{F}}\langle V(\boldsymbol{v} \cdot \boldsymbol{\nu}), \boldsymbol{u} \cdot \boldsymbol{\nu}\rangle+\rho_{\mathrm{F}}\left\langle\left(\frac{1}{2} I-K\right) \eta_{h}, \boldsymbol{u} \cdot \boldsymbol{\nu}\right\rangle,
$$

with $\eta_{h} \in \mathcal{Q}_{h}$ being the solution of

$$
\left\langle\zeta_{h}, W \eta_{h}\right\rangle=\left\langle\left(\frac{1}{2} I-K\right) \zeta_{h}, \boldsymbol{v} \cdot \boldsymbol{\nu}\right\rangle \quad \forall \zeta_{h} \in \mathcal{Q}_{h}
$$

The first terms in $b_{h}(\boldsymbol{u}, \boldsymbol{v})$ and $b_{h}(\boldsymbol{v}, \boldsymbol{u})$ clearly coincide. So do the second ones because of the symmetry of $\langle V \cdot, \cdot\rangle$. Regarding the third ones we have

$$
\left\langle\left(\frac{1}{2} I-K\right) \varphi_{h}, \boldsymbol{v} \cdot \boldsymbol{\nu}\right\rangle=\left\langle\varphi_{h}, W \eta_{h}\right\rangle=\left\langle\eta_{h}, W \varphi_{h}\right\rangle=\left\langle\left(\frac{1}{2} I-K\right) \eta_{h}, \boldsymbol{u} \cdot \boldsymbol{\nu}\right\rangle,
$$

where we have used the definitions of $\varphi_{h}$ and $\eta_{h}$ above, and the symmetry of $\langle\cdot, W \cdot\rangle$.

Let $T_{h}: \mathcal{V} \longrightarrow \mathcal{V}$ be the bounded linear operator given by

$$
T_{h} \boldsymbol{f}=\boldsymbol{u}_{h} \in \mathcal{V}_{h}: \quad a\left(\boldsymbol{u}_{h}, \boldsymbol{v}_{h}\right)=b_{h}\left(\boldsymbol{f}, \boldsymbol{v}_{h}\right) \quad \forall \boldsymbol{v}_{h} \in \mathcal{V}_{h}
$$

Because of the symmetry of $a$ and $b_{h}, T_{h}$ is self-adjoint with respect to both bilinear forms; thus all of its eigenvalues are real and positive. Furthermore, clearly $\left(\lambda_{h}, \boldsymbol{u}_{h}\right)$ is an eigenpair of $T_{h}$ if and only if $\omega_{h}:=\frac{1}{\sqrt{\lambda_{h}}}$ and $\boldsymbol{u}_{h}$ are solution of (3.1)-(3.2).

Our next goal is to prove that $T_{h}$ converges in norm to $T$, a property that will be used below to obtain spectral approximation results. Since the bilinear forms $b$ and $b_{h}$ do not coincide, (3.3) can be seen as a nonconforming finite element approximation of (2.20). In the two following lemmas we will prove consistency and approximation estimates, as usual for this kind of methods.

Lemma 3.1. There exist constants $C>0$ and $s \in\left(\frac{1}{2}, 1\right]$ such that, $\forall \boldsymbol{f}, \boldsymbol{g} \in \mathcal{V}$,

$$
\left|b(\boldsymbol{f}, \boldsymbol{g})-b_{h}(\boldsymbol{f}, \boldsymbol{g})\right| \leq C h^{s}|\boldsymbol{f}|\|\boldsymbol{g}\|_{\mathrm{H}^{1}\left(\Omega_{\mathrm{S}}\right)^{2}},
$$

and

$$
\left|b(\boldsymbol{f}, \boldsymbol{g})-b_{h}(\boldsymbol{f}, \boldsymbol{g})\right| \leq C h^{2 s}\|\boldsymbol{f}\|_{\mathrm{H}^{1}\left(\Omega_{\mathrm{S}}\right)^{2}}\|\boldsymbol{g}\|_{\mathrm{H}^{1}\left(\Omega_{\mathrm{S}}\right)^{2}} .
$$

Proof. Let $\boldsymbol{f}, \boldsymbol{g} \in \mathcal{V}$. According to the definitions of $b$ and $b_{h}$ we have

$$
b(\boldsymbol{f}, \boldsymbol{g})-b_{h}(\boldsymbol{f}, \boldsymbol{g})=\rho_{\mathrm{F}}\left[\left\langle\left(\frac{1}{2} I-K\right) \varphi, \boldsymbol{g} \cdot \boldsymbol{\nu}\right\rangle-\left\langle\left(\frac{1}{2} I-K\right) \varphi_{h}, \boldsymbol{g} \cdot \boldsymbol{\nu}\right\rangle\right],
$$

where $\varphi \in \mathcal{Q}$ and $\varphi_{h} \in \mathcal{Q}_{h}$ are respectively defined by

$$
\langle\psi, W \varphi\rangle=\left\langle\left(\frac{1}{2} I-K\right) \psi, \boldsymbol{f} \cdot \boldsymbol{\nu}\right\rangle \quad \forall \psi \in \mathcal{Q}
$$

and

Now, let $\xi \in \mathcal{Q}$ and $\xi_{h} \in \mathcal{Q}_{h}$ be the respective solutions of

$$
\left\langle\psi_{h}, W \varphi_{h}\right\rangle=\left\langle\left(\frac{1}{2} I-K\right) \psi_{h}, \boldsymbol{f} \cdot \boldsymbol{\nu}\right\rangle \quad \forall \psi_{h} \in \mathcal{Q}_{h} .
$$

$$
\langle\xi, W \psi\rangle=\left\langle\left(\frac{1}{2} I-K\right) \psi, \boldsymbol{g} \cdot \boldsymbol{\nu}\right\rangle \quad \forall \psi \in \mathcal{Q}
$$


and

$$
\left\langle\xi_{h}, W \psi_{h}\right\rangle=\left\langle\left(\frac{1}{2} I-K\right) \psi_{h}, \boldsymbol{g} \cdot \boldsymbol{\nu}\right\rangle \quad \forall \psi_{h} \in \mathcal{Q}_{h} .
$$

Then, taking $\psi=\varphi$ and $\psi_{h}=\varphi_{h}$ in the two equations above we obtain

$$
b(\boldsymbol{f}, \boldsymbol{g})-b_{h}(\boldsymbol{f}, \boldsymbol{g})=\rho_{\mathrm{F}}\left[\langle\xi, W \varphi\rangle-\left\langle\xi_{h}, W \varphi_{h}\right\rangle\right]=\rho_{\mathrm{F}}\left\langle\xi-\xi_{h}, W \varphi\right\rangle,
$$

the latter because $\left\langle\xi_{h}, W \varphi_{h}\right\rangle=\left\langle\xi_{h}, W \varphi\right\rangle$. Moreover, since $\left\langle\xi-\xi_{h}, W \varphi_{h}\right\rangle=0$, we have

$$
\left|b(\boldsymbol{f}, \boldsymbol{g})-b_{h}(\boldsymbol{f}, \boldsymbol{g})\right|=\left|\rho_{\mathrm{F}}\left\langle\xi-\xi_{h}, W\left(\varphi-\varphi_{h}\right)\right\rangle\right| \leq C\left\|\xi-\xi_{h}\right\|_{\mathrm{H}^{1 / 2}\left(\Gamma_{\mathrm{I}}\right)}\left\|\varphi-\varphi_{h}\right\|_{\mathrm{H}^{1 / 2}\left(\Gamma_{\mathrm{I}}\right)} .
$$

To estimate $\left\|\varphi-\varphi_{h}\right\|_{\mathrm{H}^{1 / 2}\left(\Gamma_{\mathrm{I}}\right)}$, let us recall that $\varphi=\left.z\right|_{\Gamma_{\mathrm{I}}}$, where $z \in \mathrm{H}^{1}\left(\Omega_{\mathrm{F}}\right)$ is a solution of the compatible Neumann problem (see for instance [17])

$$
\begin{aligned}
\Delta z=0 & \text { in } \Omega_{\mathrm{F}}, \\
\frac{\partial z}{\partial \nu}=\boldsymbol{f} \cdot \boldsymbol{\nu} & \text { on } \Gamma_{\mathrm{I}} .
\end{aligned}
$$

Then the trace theorem, the Lax-Milgram lemma, and Lemma 2.7 yield

$$
\|\varphi\|_{\mathrm{H}^{1 / 2}\left(\Gamma_{\mathrm{I}}\right)} \leq C\|\boldsymbol{f} \cdot \boldsymbol{\nu}\|_{\mathrm{H}^{-1 / 2}\left(\Gamma_{\mathrm{I}}\right)} \leq C|\boldsymbol{f}| .
$$

Moreover, since $\boldsymbol{f} \cdot \boldsymbol{\nu} \in \mathrm{H}^{1 / 2}\left(\Gamma_{j}\right)$ for all the edges $\Gamma_{j}$ of $\Gamma_{\mathrm{I}}(j=1, \ldots, J)$, we know that $\exists s \in\left(\frac{1}{2}, 1\right]$ such that $z \in \mathrm{H}^{1+s}\left(\Omega_{\mathrm{F}}\right)$ (see [11]), and

$$
\|z\|_{\mathrm{H}^{1+s}\left(\Omega_{\mathrm{F}}\right)} \leq C \sum_{j=1}^{J}\|\boldsymbol{f} \cdot \boldsymbol{\nu}\|_{\mathrm{H}^{1 / 2}\left(\Gamma_{j}\right)} .
$$

Thus, $\varphi=\left.z\right|_{\Gamma_{\mathrm{I}}} \in \mathrm{H}^{1 / 2+s}\left(\Gamma_{\mathrm{I}}\right)$. Therefore, its Lagrange interpolant $\varphi^{\mathrm{I}} \in \mathcal{Q}_{h}$ is well defined and satisfies

$$
\left\|\varphi-\varphi^{\mathrm{I}}\right\|_{\mathrm{H}^{1 / 2}\left(\Gamma_{\mathrm{I}}\right)} \leq h^{s}\|\varphi\|_{\mathrm{H}^{1 / 2+s}\left(\Gamma_{\mathrm{I}}\right)} \leq C h^{s}\|z\|_{\mathrm{H}^{1+s}\left(\Omega_{\mathrm{F}}\right)} \leq C h^{s} \sum_{j=1}^{J}\|\boldsymbol{f} \cdot \boldsymbol{\nu}\|_{\mathrm{H}^{1 / 2}\left(\Gamma_{\mathrm{I}}\right)} .
$$

Then, by Céa's lemma, we have

$$
\left\|\varphi-\varphi_{h}\right\|_{\mathrm{H}^{1 / 2}\left(\Gamma_{\mathrm{I}}\right)} \leq C\left\|\varphi-\varphi^{\mathrm{I}}\right\|_{\mathrm{H}^{1 / 2}\left(\Gamma_{\mathrm{I}}\right)} \leq C h^{s} \sum_{j=1}^{J}\|\boldsymbol{f} \cdot \boldsymbol{\nu}\|_{\mathrm{H}^{1 / 2}\left(\Gamma_{\mathrm{I}}\right)} \leq C h^{s}\|\boldsymbol{f}\|_{\mathrm{H}^{1}\left(\Omega_{\mathrm{S}}\right)^{2}} .
$$

Proceeding in the same way we obtain an analogous estimate for $\left\|\xi-\xi_{h}\right\|_{\mathrm{H}^{1 / 2}\left(\Gamma_{\mathrm{I}}\right)}$; namely,

$$
\left\|\xi-\xi_{h}\right\|_{\mathrm{H}^{1 / 2}\left(\Gamma_{\mathrm{I}}\right)} \leq C h^{s}\|\boldsymbol{g}\|_{\mathrm{H}^{1}\left(\Omega_{\mathrm{S}}\right)^{2}} .
$$

Hence, (3.5) follows from these two estimates and (3.7).

On the other hand, to prove (3.4), we use (3.6), the estimate above, and (3.8) to obtain

$$
\left|b(\boldsymbol{f}, \boldsymbol{g})-b_{h}(\boldsymbol{f}, \boldsymbol{g})\right| \leq C\left\|\xi-\xi_{h}\right\|_{\mathrm{H}^{1 / 2}\left(\Gamma_{\mathrm{I}}\right)}\|\varphi\|_{\mathrm{H}^{1 / 2}\left(\Gamma_{\mathrm{I}}\right)} \leq C h^{s}\|\boldsymbol{g}\|_{\mathrm{H}^{1}\left(\Omega_{\mathrm{S}}\right)^{2}}|\boldsymbol{f}|,
$$

which completes the proof.

Remark 3.2. The constant $s \in\left(\frac{1}{2}, 1\right]$ of the previous lemma is $s=1$, if $\Omega_{\mathrm{F}}$ is convex, and any $s<\frac{\pi}{\theta}$, with $\theta$ being the largest reentrant angle of $\Omega_{\mathrm{F}}$, otherwise (see [11]). 
Lemma 3.3. Let $\boldsymbol{u} \in \mathcal{V} \cap \mathrm{H}^{1+t}\left(\Omega_{\mathrm{S}}\right)^{2}$, with $t \in(0,1]$. Then, there exists $\hat{\boldsymbol{u}} \in \mathcal{V}_{h}$ and a constant $C>0$, independent of $\boldsymbol{u}$, such that

$$
\|\boldsymbol{u}-\hat{\boldsymbol{u}}\|_{\mathrm{H}^{1}\left(\Omega_{\mathrm{S}}\right)^{2}} \leq C h^{t}\|\boldsymbol{u}\|_{\mathrm{H}^{1+t}\left(\Omega_{\mathrm{S}}\right)^{2}} .
$$

Proof. Since $\boldsymbol{u} \in \mathrm{H}^{1+t}\left(\Omega_{\mathrm{S}}\right)^{2}$ with $t>0$, its Lagrange interpolant $\boldsymbol{u}^{\mathrm{I}} \in \mathcal{V}_{h}$ is well defined and satisfies

$$
\left\|\boldsymbol{u}-\boldsymbol{u}^{\mathrm{I}}\right\|_{\mathrm{H}^{1}\left(\Omega_{\mathrm{S}}\right)^{2}} \leq C h^{t}\|\boldsymbol{u}\|_{\mathrm{H}^{1+t}\left(\Omega_{\mathrm{S}}\right)^{2}} \text {. }
$$

However, in general, $\int_{\partial \Omega_{\mathrm{S}}} \boldsymbol{u}^{\mathrm{I}} \cdot \boldsymbol{\nu} \mathrm{d} s \neq \int_{\partial \Omega_{\mathrm{S}}} \boldsymbol{u} \cdot \boldsymbol{\nu} \mathrm{d} s$. Then we define $\hat{\boldsymbol{u}}:=\boldsymbol{u}^{\mathrm{I}}-c \boldsymbol{u}_{0}$, where $\boldsymbol{u}_{0}$ is a fixed vector field in $\mathcal{L}_{h}\left(\Omega_{\mathrm{S}}\right)^{2}$ satisfying $\left.\boldsymbol{u}_{0}\right|_{\Gamma_{\mathrm{D}}}=0$ and $\int_{\Gamma_{\mathrm{I}}} \boldsymbol{u}_{0} \cdot \boldsymbol{\nu} \mathrm{d} s=1$, and $c$ is chosen such that $\int_{\Gamma_{\mathrm{I}}} \hat{\boldsymbol{u}} \cdot \boldsymbol{\nu} \mathrm{d} s=0$ holds true; namely,

$$
c=\int_{\Gamma_{\mathrm{I}}} \boldsymbol{u}^{\mathrm{I}} \cdot \boldsymbol{\nu} \mathrm{d} s
$$

Therefore

$$
\|\boldsymbol{u}-\hat{\boldsymbol{u}}\|_{\mathrm{H}^{1}\left(\Omega_{\mathrm{S}}\right)^{2}} \leq\left\|\boldsymbol{u}-\boldsymbol{u}^{\mathrm{I}}\right\|_{\mathrm{H}^{1}\left(\Omega_{\mathrm{S}}\right)^{2}}+\left\|c \boldsymbol{u}_{0}\right\|_{\mathrm{H}^{1}\left(\Omega_{\mathrm{S}}\right)^{2}} .
$$

Now, since $\int_{\Gamma_{\mathrm{I}}} \boldsymbol{u} \cdot \boldsymbol{\nu} \mathrm{d} s=0$ because $\boldsymbol{u} \in \mathcal{V}$, we obtain

$$
\begin{aligned}
\left\|c \boldsymbol{u}_{0}\right\|_{\mathrm{H}^{1}\left(\Omega_{\mathrm{S}}\right)^{2}} & =|c|\left\|\boldsymbol{u}_{0}\right\|_{\mathrm{H}^{1}\left(\Omega_{\mathrm{S}}\right)^{2}}=\left|\int_{\Gamma_{\mathrm{I}}}\left(\boldsymbol{u}^{\mathrm{I}} \cdot \boldsymbol{\nu}-\boldsymbol{u} \cdot \boldsymbol{\nu}\right) \mathrm{d} s\right|\left\|\boldsymbol{u}_{0}\right\|_{\mathrm{H}^{1}\left(\Omega_{\mathrm{S}}\right)^{2}} \\
& \leq C\left\|\boldsymbol{u}^{\mathrm{I}} \cdot \boldsymbol{\nu}-\boldsymbol{u} \cdot \boldsymbol{\nu}\right\|_{\mathrm{L}^{2}\left(\Gamma_{\mathrm{I}}\right)} \leq C h^{\min \left\{1, \frac{1}{2}+t\right\}}\|\boldsymbol{u} \cdot \boldsymbol{\nu}\|_{\mathrm{H}^{1 / 2+t}\left(\Gamma_{\mathrm{I}}\right)} \leq C h^{t}\|\boldsymbol{u}\|_{\mathrm{H}^{1+t}\left(\Omega_{\mathrm{S}}\right)^{2}} .
\end{aligned}
$$

Thus, we conclude the proof from the three estimates above.

As a consequence of the last two lemmas, we can prove now the convergence in norm of the discrete operators.

Lemma 3.4. Let $T$ and $T_{h}$ be the operators defined by (2.20) and (3.3), respectively. Then, there exists a constant $C>0$, such that

where $t \in(0,1]$ is as in Lemma 2.8 .

$$
\left\|\left(T-T_{h}\right) \boldsymbol{f}\right\|_{\mathrm{H}^{1}\left(\Omega_{\mathrm{S}}\right)^{2}} \leq C h^{t}\|\boldsymbol{f}\|_{\mathrm{H}^{1}\left(\Omega_{\mathrm{S}}\right)^{2}} \quad \forall \boldsymbol{f} \in \mathcal{V}
$$

Proof. For $\boldsymbol{f} \in \mathcal{V}$, let $\boldsymbol{u}=T \boldsymbol{f}$ and $\boldsymbol{u}_{h}=T_{h} \boldsymbol{f}$. From Strang's lemma (see, for instance, [7]) there holds

$$
\left\|\boldsymbol{u}-\boldsymbol{u}_{h}\right\|_{\mathrm{H}^{1}\left(\Omega_{\mathrm{S}}\right)^{2}} \leq C\left[\inf _{\hat{\boldsymbol{u}} \in \mathcal{V}_{h}}\|\boldsymbol{u}-\hat{\boldsymbol{u}}\|_{\mathrm{H}^{1}\left(\Omega_{\mathrm{S}}\right)^{2}}+\sup _{\boldsymbol{v}_{h} \in \mathcal{V}_{h}} \frac{\left|b\left(\boldsymbol{f}, \boldsymbol{v}_{h}\right)-b_{h}\left(\boldsymbol{f}, \boldsymbol{v}_{h}\right)\right|}{\left\|\boldsymbol{v}_{h}\right\|_{\mathrm{H}^{1}\left(\Omega_{\mathrm{S}}\right)^{2}}}\right] .
$$

Therefore, the result follows from (3.5), Lemmas 3.3 and 2.8 , and the inequalities $|\boldsymbol{f}| \leq C\|\boldsymbol{f}\|_{\mathrm{H}^{1}\left(\Omega_{\mathrm{S}}\right)^{2}}$ and $t \leq 1<2 s$.

Remark 3.5. The following estimate, which will be used in Theorem 3.7 below, is obtained by using (3.4) instead of (3.5) in the proof of the previous lemma:

$$
\left\|\left(T-T_{h}\right) \boldsymbol{f}\right\|_{\mathrm{H}^{1}\left(\Omega_{\mathrm{S}}\right)^{2}} \leq C h^{\min \{s, t\}}|\boldsymbol{f}| \quad \forall \boldsymbol{f} \in \mathcal{V}
$$

From the above lemma we know that $T_{h}$ converges to $T$ in $\mathrm{H}^{1}\left(\Omega_{\mathrm{S}}\right)^{2}$ norm and, consequently, the isolated parts of the spectrum of $T$ are approximated by isolated parts of the spectrum of $T_{h}$. In other words, given any eigenvalue $\lambda$ of $T$ of multiplicity $m$, there are exactly $m$ eigenvalues $\lambda_{h}^{(1)}, \ldots, \lambda_{h}^{(m)}$ of $T_{h}$ (repeated according to their respective multiplicities) converging to $\lambda$ as $h$ goes to zero.

Let $\lambda$ be a positive fixed eigenvalue of $T$ of multiplicity $m$ and let $\mathcal{E}$ be its associated eigenspace. Let $\mathcal{E}_{h}$ denote the direct sum of the corresponding eigenspaces of the $m$ eigenvalues $\lambda_{h}^{(1)}, \ldots, \lambda_{h}^{(m)}$ of $T_{h}$ that converge to $\lambda$. 
Thus, by applying the spectral approximation theory for compact operators as stated in [2] (Th. 7.1) and by using the previous lemma we obtain the following error estimates:

Theorem 3.6. There exists a strictly positive constant $C$, independent of $h$, such that

$$
\inf _{\boldsymbol{v}_{h} \in \mathcal{E}_{h}}\left\|\boldsymbol{u}-\boldsymbol{v}_{h}\right\|_{\mathrm{H}^{1}\left(\Omega_{\mathrm{S}}\right)^{2}} \leq C h^{t}\|\boldsymbol{u}\|_{\mathrm{H}^{1}\left(\Omega_{\mathrm{S}}\right)^{2}} \quad \forall \boldsymbol{u} \in \mathcal{E},
$$

and

$$
\inf _{\boldsymbol{v} \in \mathcal{E}}\left\|\boldsymbol{u}_{h}-\boldsymbol{v}\right\|_{\mathrm{H}^{1}\left(\Omega_{\mathrm{S}}\right)^{2}} \leq C h^{t}\left\|\boldsymbol{u}_{h}\right\|_{\mathrm{H}^{1}\left(\Omega_{\mathrm{S}}\right)^{2}} \quad \forall \boldsymbol{u}_{h} \in \mathcal{E}_{h},
$$

where $t \in(0,1]$ is as in Lemma 2.8.

Now, let $\mathcal{H}$ denote the Hilbert space obtained as the completion of $\mathcal{V}$ with respect to the norm $|\cdot|$. Then $(\mathcal{V},\|\cdot\|)$ is continuously and densely included in $(\mathcal{H},|\cdot|)$. Thus, $T$ can be uniquely extended to $\mathcal{H}$ and this extension is also self-adjoint with respect to $b$. Analogously, we can extend $T_{h}$ to $\mathcal{H}$, which, however, does not become self-adjoint with respect to $b$. In fact, let us call $T_{h}^{*}$ its adjoint with respect to this inner product, i.e.,

$$
b\left(T_{h}^{*} \boldsymbol{v}, \boldsymbol{w}\right)=b\left(\boldsymbol{v}, T_{h} \boldsymbol{w}\right) \quad \forall \boldsymbol{v}, \boldsymbol{w} \in \mathcal{H} .
$$

Hence, we have

$$
b\left(T_{h}^{*} \boldsymbol{v}, \boldsymbol{w}\right)=a\left(T \boldsymbol{v}, T_{h} \boldsymbol{w}\right) \quad \forall \boldsymbol{v}, \boldsymbol{w} \in \mathcal{H},
$$

whereas

$$
b\left(T_{h} \boldsymbol{v}, \boldsymbol{w}\right)=b\left(\boldsymbol{w}, T_{h} \boldsymbol{v}\right)=a\left(T \boldsymbol{w}, T_{h} \boldsymbol{v}\right)=a\left(T_{h} \boldsymbol{v}, T \boldsymbol{w}\right) \quad \forall \boldsymbol{v}, \boldsymbol{w} \in \mathcal{H},
$$

which, in general, do not coincide. Nevertheless, a larger order of convergence can be proved for the approximation of the eigenvalues:

Theorem 3.7. There exists a strictly positive constant $C$, independent of $h$, such that

$$
\left|\lambda-\lambda_{h}^{(i)}\right| \leq C h^{2 r}, \quad i=1, \ldots, m,
$$

where $r:=\min \{s, t\}$, with $s \in\left(\frac{1}{2}, 1\right]$ as in Remark 3.2 and $t \in(0,1]$ as in Lemma 2.8.

Proof. By applying Theorem 7.3 in [2] to our situation, we obtain

$$
\left|\lambda-\lambda_{h}^{(i)}\right| \leq C\left[\sup _{\substack{\boldsymbol{f} \in \mathcal{E} \\|\boldsymbol{f}|=1}} \sup _{\substack{\boldsymbol{g} \in \mathcal{E}|=1\\| \boldsymbol{g} \mid=1}}\left|b\left(\left(T-T_{h}\right) \boldsymbol{f}, \boldsymbol{g}\right)\right|+\left.\left|\left(T-T_{h}\right)\right|_{\mathcal{E}}||\left(T-T_{h}^{*}\right)\right|_{\mathcal{E}} \mid\right], \quad i=1, \ldots, m .
$$

Let $\boldsymbol{f}, \boldsymbol{g} \in \mathcal{E}$ and let

$$
\boldsymbol{u}=T \boldsymbol{f} \in \mathcal{V}, \quad \boldsymbol{v}=T \boldsymbol{g} \in \mathcal{V}, \quad \boldsymbol{u}_{h}=T_{h} \boldsymbol{f} \in \mathcal{V}_{h}, \quad \text { and } \quad \boldsymbol{v}_{h}=T_{h} \boldsymbol{g} \in \mathcal{V}_{h}
$$

Note that, for $\boldsymbol{f} \in \mathcal{E}, \boldsymbol{u}=T \boldsymbol{f}=\lambda \boldsymbol{f}$, then $\boldsymbol{f}=\frac{1}{\lambda} \boldsymbol{u}$ and, by Lemma 2.8,

$$
\|\boldsymbol{f}\|_{\mathrm{H}^{1}\left(\Omega_{\mathrm{S}}\right)^{2}} \leq\|\boldsymbol{f}\|_{\mathrm{H}^{1+t}\left(\Omega_{\mathrm{S}}\right)^{2}}=\frac{1}{\lambda}\|\boldsymbol{u}\|_{\mathrm{H}^{1+t}\left(\Omega_{\mathrm{S}}\right)^{2}} \leq C|\boldsymbol{f}| .
$$

Analogously, for $\boldsymbol{g} \in \mathcal{E},\|\boldsymbol{g}\|_{\mathrm{H}^{1}\left(\Omega_{\mathrm{S}}\right)^{2}} \leq C|\boldsymbol{g}|$.

Now, for the first term in the right-hand side of (3.9), we have

$$
b\left(\left(T-T_{h}\right) \boldsymbol{f}, \boldsymbol{g}\right)=b\left(\boldsymbol{u}-\boldsymbol{u}_{h}, \boldsymbol{g}\right)=a\left(\boldsymbol{u}-\boldsymbol{u}_{h}, \boldsymbol{v}\right)=a\left(\boldsymbol{u}-\boldsymbol{u}_{h}, \boldsymbol{v}-\boldsymbol{v}_{h}\right)+a\left(\boldsymbol{u}-\boldsymbol{u}_{h}, \boldsymbol{v}_{h}\right) .
$$


From Lemma 3.4,

$$
a\left(\boldsymbol{u}-\boldsymbol{u}_{h}, \boldsymbol{v}-\boldsymbol{v}_{h}\right) \leq C\left\|\boldsymbol{u}-\boldsymbol{u}_{h}\right\|_{\mathrm{H}^{1}\left(\Omega_{\mathrm{S}}\right)^{2}}\left\|\boldsymbol{v}-\boldsymbol{v}_{h}\right\|_{\mathrm{H}^{1}\left(\Omega_{\mathrm{S}}\right)^{2}} \leq C h^{2 t}\|\boldsymbol{f}\|_{\mathrm{H}^{1}\left(\Omega_{\mathrm{S}}\right)^{2}}\|\boldsymbol{g}\|_{\mathrm{H}^{1}\left(\Omega_{\mathrm{S}}\right)^{2}}
$$

and, consequently,

$$
a\left(\boldsymbol{u}-\boldsymbol{u}_{h}, \boldsymbol{v}-\boldsymbol{v}_{h}\right) \leq C h^{2 t}|\boldsymbol{f}||\boldsymbol{g}|
$$

On the other hand, from $(3.5)$

$$
a\left(\boldsymbol{u}-\boldsymbol{u}_{h}, \boldsymbol{v}_{h}\right)=b\left(\boldsymbol{f}, \boldsymbol{v}_{h}\right)-b_{h}\left(\boldsymbol{f}, \boldsymbol{v}_{h}\right) \leq C h^{2 s}\|\boldsymbol{f}\|_{\mathrm{H}^{1}\left(\Omega_{\mathrm{S}}\right)^{2}}\left\|\boldsymbol{v}_{h}\right\|_{\mathrm{H}^{1}\left(\Omega_{\mathrm{S}}\right)^{2}} .
$$

Now, since from Lemma 3.4,

$$
\left\|\boldsymbol{v}_{h}\right\|_{\mathrm{H}^{1}\left(\Omega_{\mathrm{S}}\right)^{2}} \leq\|\boldsymbol{v}\|_{\mathrm{H}^{1}\left(\Omega_{\mathrm{S}}\right)^{2}}+\left\|\boldsymbol{v}-\boldsymbol{v}_{h}\right\|_{\mathrm{H}^{1}\left(\Omega_{\mathrm{S}}\right)^{2}} \leq C\|\boldsymbol{g}\|_{\mathrm{H}^{1}\left(\Omega_{\mathrm{S}}\right)^{2}}+C h^{t}\|\boldsymbol{g}\|_{\mathrm{H}^{1}\left(\Omega_{\mathrm{S}}\right)^{2}} \leq C\|\boldsymbol{g}\|_{\mathrm{H}^{1}\left(\Omega_{\mathrm{S}}\right)^{2}}
$$

there holds

$$
a\left(\boldsymbol{u}-\boldsymbol{u}_{h}, \boldsymbol{v}_{h}\right) \leq C h^{2 s}|\boldsymbol{f}||\boldsymbol{g}|
$$

Hence,

$$
\sup _{\substack{\boldsymbol{f} \in \mathcal{E} \\|\boldsymbol{f}|=1}} \sup _{\substack{\boldsymbol{g} \in \mathcal{E} \\|\boldsymbol{g}|=1}}\left|b\left(\left(T-T_{h}\right) \boldsymbol{f}, \boldsymbol{g}\right)\right| \leq C h^{\min \{2 s, 2 t\}} .
$$

For the second term in the right-hand side of (3.9) we have $\left|\left(T-T_{h}\right)\right|_{\mathcal{E}} \mid \leq C h^{\min \{s, t\}}$. Indeed, from Lemma 3.4, for $\boldsymbol{f} \in \mathcal{E}$ we have

$$
\left|\left(T-T_{h}\right) \boldsymbol{f}\right| \leq C\left\|\left(T-T_{h}\right) \boldsymbol{f}\right\|_{\mathrm{H}^{1}\left(\Omega_{\mathrm{S}}\right)^{2}} \leq C h^{t}\|\boldsymbol{f}\|_{\mathrm{H}^{1}\left(\Omega_{\mathrm{S}}\right)^{2}} \leq C h^{t}|\boldsymbol{f}| .
$$

To conclude the theorem, we have to estimate the term $\left|\left(T-T_{h}^{*}\right)\right|_{\mathcal{E}} \mid$. Since $\mathcal{V}$ is dense in $\mathcal{H}$, we have

$$
\left|\left(T-T_{h}^{*}\right)\right|_{\mathcal{E}}\left|=\sup _{\substack{\boldsymbol{g} \in \mathcal{E} \\|\boldsymbol{g}|=1}}\right|\left(T-T_{h}^{*}\right) \boldsymbol{g} \mid=\sup _{\substack{\boldsymbol{g} \in \mathcal{E} \\|\boldsymbol{g}|=1}} \sup _{\substack{\tilde{\boldsymbol{f}} \in \mathcal{V} \\|\tilde{\boldsymbol{f}}|=1}} b\left(\left(T-T_{h}^{*}\right) \boldsymbol{g}, \tilde{\boldsymbol{f}}\right)=\sup _{\substack{\boldsymbol{g} \in \mathcal{E} \\|\boldsymbol{g}|=1}} \sup _{\substack{\tilde{\boldsymbol{f}} \in \mathcal{V} \\|\tilde{\boldsymbol{f}}|=1}} b\left(\left(T-T_{h}\right) \tilde{\boldsymbol{f}}, \boldsymbol{g}\right) .
$$

Let $\boldsymbol{g} \in \mathcal{E}$ and $\tilde{\boldsymbol{f}} \in \mathcal{V}$. We proceed as above, but taking into account that (3.10) does not necessarily hold for $\tilde{\boldsymbol{f}} \notin \mathcal{E}$. Then, we use Remark 3.5 instead of Lemma 3.4 for $\tilde{\boldsymbol{f}}$ and (3.4) instead of (3.5). Thus, we obtain

$$
b\left(\left(T-T_{h}\right) \tilde{\boldsymbol{f}}, \boldsymbol{g}\right) \leq C h^{\min \{s, 2 t\}}|\tilde{\boldsymbol{f}}|\|\boldsymbol{g}\|_{\mathrm{H}^{1}\left(\Omega_{\mathrm{S}}\right)^{2}} \leq C h^{\min \{s, 2 t\}}|\tilde{\boldsymbol{f}}||\boldsymbol{g}|
$$

Then, $\left|\left(T-T_{h}^{*}\right)\right|_{\mathcal{E}} \mid \leq C h^{\min \{s, t\}}$, which together with the estimates of the other two terms allow us to conclude the proof.

\section{NumericAl RESUltS}

We begin by providing an equivalent formulation of problem (3.1)-(3.2) which avoids imposing the constraint $\int_{\Gamma_{\mathrm{I}}} \boldsymbol{v}_{h} \cdot \boldsymbol{\nu} \mathrm{d} s=0$ on the space $\mathcal{V}_{h}$. In fact, let $\mathcal{W}_{h}$ and $\mathcal{P}_{h}$ be the spaces defined by

$$
\mathcal{W}_{h}:=\left\{\boldsymbol{v}_{h} \in \mathcal{L}_{h}\left(\Omega_{\mathrm{S}}\right)^{2}:\left.\boldsymbol{v}_{h}\right|_{\Gamma_{\mathrm{D}}}=0\right\}
$$

and

$$
\mathcal{P}_{h}:=\mathcal{L}_{h}\left(\Gamma_{\mathrm{I}}\right)
$$


Then, proceeding similarly as in the proof of Lemma 2.4 one can show that (3.1)-(3.2) is equivalent to the following problem:

Find $\omega_{h} \geq 0$ and $\mathbf{0} \neq\left(\boldsymbol{u}_{h}, \varphi_{h}\right) \in \mathcal{W}_{h} \times \mathcal{P}_{h}$ such that

$$
\int_{\Omega_{\mathrm{S}}} \boldsymbol{\sigma}\left(\boldsymbol{u}_{h}\right): \boldsymbol{\varepsilon}\left(\boldsymbol{v}_{h}\right) \mathrm{d} x=\omega_{h}^{2}\left[\int_{\Omega_{\mathrm{S}}} \rho_{\mathrm{S}} \boldsymbol{u}_{h} \cdot \boldsymbol{v}_{h} \mathrm{~d} x+\rho_{\mathrm{F}}\left\langle V\left(\boldsymbol{u}_{h} \cdot \boldsymbol{\nu}\right), \boldsymbol{v}_{h} \cdot \boldsymbol{\nu}\right\rangle+\rho_{\mathrm{F}}\left\langle\left(\frac{1}{2} I-K\right) \varphi_{h}, \boldsymbol{v}_{h} \cdot \boldsymbol{\nu}\right\rangle\right]_{\forall \boldsymbol{v}_{h} \in \mathcal{W}_{h}}
$$

and

$$
\left\langle\psi_{h}, W \varphi_{h}\right\rangle=\left\langle\left(\frac{1}{2} I-K\right) \psi_{h}, \boldsymbol{u}_{h} \cdot \boldsymbol{\nu}\right\rangle \quad \forall \psi_{h} \in \mathcal{P}_{h} .
$$

To obtain a symmetric form of this problem, we add the second equation times $\omega_{h}^{2} \rho_{\mathrm{F}}$ to the first one. Thus we obtain the following formulation which is clearly equivalent to the previous one for $\omega_{h} \neq 0$ :

Find $\omega_{h} \geq 0$ and $\mathbf{0} \neq\left(\boldsymbol{u}_{h}, \varphi_{h}\right) \in \mathcal{W}_{h} \times \mathcal{P}_{h}$ such that

$$
\begin{aligned}
\int_{\Omega_{\mathrm{S}}} \boldsymbol{\sigma}\left(\boldsymbol{u}_{h}\right): \varepsilon\left(\boldsymbol{v}_{h}\right) \mathrm{d} x=\omega_{h}^{2}[ & \int_{\Omega_{\mathrm{S}}} \rho_{\mathrm{S}} \boldsymbol{u}_{h} \cdot \boldsymbol{v}_{h} \mathrm{~d} x+\rho_{\mathrm{F}}\left\langle V\left(\boldsymbol{u}_{h} \cdot \boldsymbol{\nu}\right), \boldsymbol{v}_{h} \cdot \boldsymbol{\nu}\right\rangle+\rho_{\mathrm{F}}\left\langle\left(\frac{1}{2} I-K\right) \varphi_{h}, \boldsymbol{v}_{h} \cdot \boldsymbol{\nu}\right\rangle \\
& \left.+\rho_{\mathrm{F}}\left\langle\left(\frac{1}{2} I-K\right) \psi_{h}, \boldsymbol{u}_{h} \cdot \boldsymbol{\nu}\right\rangle-\rho_{\mathrm{F}}\left\langle\psi_{h}, W \varphi_{h}\right\rangle\right] \quad \forall\left(\boldsymbol{v}_{h}, \psi_{h}\right) \in \mathcal{W}_{h} \times \mathcal{P}_{h} .
\end{aligned}
$$

Let $\left\{\boldsymbol{u}_{1}, \ldots, \boldsymbol{u}_{n}\right\}$ and $\left\{\varphi_{1}, \ldots, \varphi_{m}\right\}$ be bases of $\mathcal{W}_{h}$ and $\mathcal{P}_{h}$, respectively. For $\boldsymbol{u}_{h} \in \mathcal{W}_{h}$ and $\varphi_{h} \in \mathcal{P}_{h}$, let $\beta_{1}, \ldots, \beta_{n}$ and $\gamma_{1}, \ldots, \gamma_{m}$ be scalars such that

$$
\boldsymbol{u}_{h}=\sum_{i=1}^{n} \beta_{i} \boldsymbol{u}_{i} \quad \text { and } \quad \varphi_{h}=\sum_{k=1}^{m} \gamma_{k} \varphi_{k}
$$

Let

$$
\begin{aligned}
& \mathbf{A}:=\left(a_{i j}\right) \in \mathbb{R}^{n \times n}, \quad \text { with } a_{i j}:=\int_{\Omega_{\mathrm{S}}} \boldsymbol{\sigma}\left(\boldsymbol{u}_{i}\right): \boldsymbol{\varepsilon}\left(\boldsymbol{u}_{j}\right) \mathrm{d} x, \quad i, j=1, \ldots, n, \\
& \mathbf{B}:=\left(b_{i j}\right) \in \mathbb{R}^{n \times n}, \quad \text { with } b_{i j}:=\int_{\Omega_{\mathrm{S}}} \rho_{\mathrm{S}} \boldsymbol{u}_{i} \cdot \boldsymbol{u}_{j} \mathrm{~d} x+\rho_{\mathrm{F}}\left\langle V\left(\boldsymbol{u}_{i} \cdot \boldsymbol{\nu}\right), \boldsymbol{u}_{j} \cdot \boldsymbol{\nu}\right\rangle, \quad i, j=1, \ldots, n, \\
& \mathbf{C}:=\left(c_{k j}\right) \in \mathbb{R}^{m \times n}, \quad \text { with } c_{k j}:=\rho_{\mathrm{F}}\left\langle\left(\frac{1}{2} I-K\right) \varphi_{k}, \boldsymbol{u}_{j} \cdot \boldsymbol{\nu}\right\rangle, \quad k=1, \ldots, m, \quad j=1, \ldots, n, \\
& \mathbf{D}:=\left(d_{k l}\right) \in \mathbb{R}^{m \times m}, \quad \text { with } d_{k l}:=\rho_{\mathrm{F}}\left\langle V \dot{\varphi}_{k}, \dot{\psi}_{l}\right\rangle, \quad k, l=1, \ldots, m,
\end{aligned}
$$

where, to avoid computing the integrals involving the hypersingular layer potential, for the entries of the last matrix we have used the following identity (see e.g. [10]):

$$
\langle\psi, W \varphi\rangle=\langle V \dot{\varphi}, \dot{\psi}\rangle \quad \forall \varphi, \psi \in \mathrm{H}^{1 / 2}\left(\Gamma_{\mathrm{I}}\right),
$$

where the dot denotes the tangential derivative along $\Gamma_{\mathrm{I}}$. 


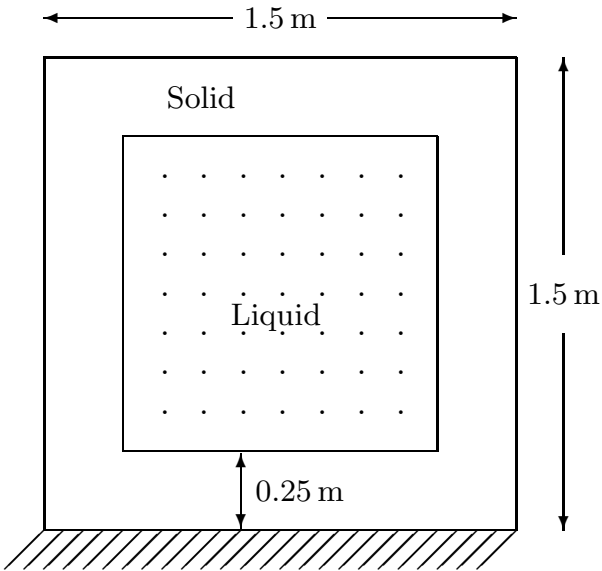

Figure 2. Geometrical data.

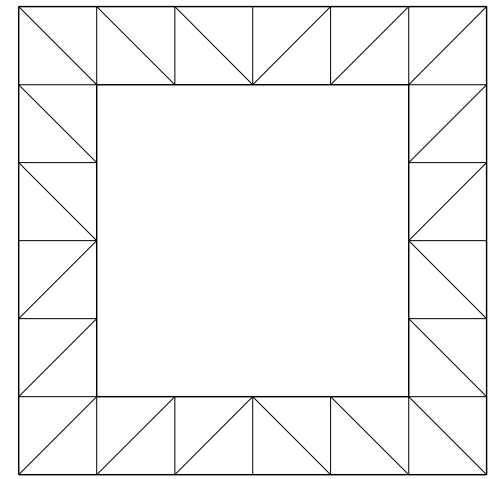

Figure 3. Initial mesh $(N=1)$.

Then the matrix form of the discrete problem (4.1) is the following:

Find $\omega_{h} \geq 0, \boldsymbol{\beta}=\left(\beta_{1}, \ldots, \beta_{n}\right) \in \mathbb{R}^{n}$, and $\boldsymbol{\gamma}=\left(\gamma_{1}, \ldots, \gamma_{m}\right) \in \mathbb{R}^{m}$, with $(\boldsymbol{\beta}, \boldsymbol{\gamma}) \neq \mathbf{0}$, such that

$$
\left(\begin{array}{cc}
\mathbf{A} & \mathbf{0} \\
\mathbf{0} & \mathbf{0}
\end{array}\right)\left(\begin{array}{l}
\boldsymbol{\beta} \\
\boldsymbol{\gamma}
\end{array}\right)=\omega_{h}^{2}\left(\begin{array}{cc}
\mathbf{B} & \mathbf{C} \\
\mathbf{C}^{\mathrm{t}} & -\mathbf{D}
\end{array}\right)\left(\begin{array}{l}
\boldsymbol{\beta} \\
\boldsymbol{\gamma}
\end{array}\right) .
$$

This is a generalized eigenvalue problem involving symmetric matrices

$$
\mathcal{A}:=\left(\begin{array}{cc}
\mathbf{A} & \mathbf{0} \\
\mathbf{0} & \mathbf{0}
\end{array}\right) \quad \text { and } \quad \mathcal{B}:=\left(\begin{array}{cc}
\mathbf{B} & \mathbf{C} \\
\mathbf{C}^{\mathrm{t}} & -\mathbf{D}
\end{array}\right) .
$$

To end this section, we report the results of a numerical test computed with the coupled BEM/FEM method described above (see [22] for more details). This test has been performed with a MATLAB ${ }^{\circledR}$ code that we have developed, in which we have used the MATLAB command sptarn to solve the algebraic eigenvalue problem (4.2). This eigensolver is based on Arnoldi method with restarting, applied to the symmetric pencil $\mathcal{A}-\sigma \mathcal{B}$.

The double integrals in the BEM terms arising in matrices $\mathbf{B}, \mathbf{C}$, and $\mathbf{D}$ have been computed with a software described in [9]. It is based on using a highly accurate quadrature formula for the outer integrals, combined with analytical integration for the inner ones. See [9] for details.

We have chosen a test example which consists of a closed steel vessel completely filled by an incompressible liquid. The geometrical data can be seen in Figure 2.

We have used the following physical parameters, which correspond to steel and water:

- solid density: $\rho_{\mathrm{S}}=7700 \mathrm{~kg} / \mathrm{m}^{3}$;

- Young modulus: $E_{\mathrm{S}}=1.4410^{11} \mathrm{~Pa}$;

- Poisson coefficient: $\nu_{\mathrm{S}}=0.35$;

- fluid density: $\rho_{\mathrm{F}}=1000 \mathrm{~kg} / \mathrm{m}^{3}$.

We have used several meshes which are successive uniform refinements of the coarse initial triangulation shown in Figure 3. The refinement parameter $N$ is the number of element layers across the thickness of the solid $(N=1$ for the mesh in Fig. 3).

Table 1 shows the frequencies of the lowest-frequency hydroelastic vibration modes of the fluid-solid coupled system computed on several meshes. The table also includes more accurate values obtained by extrapolation, as well as the computed convergence rates. Finally, we report in the last column the corresponding extrapolated values obtained with the finite element method in [6]. The last two columns show that the agreement between both methods is excellent. 
TABLE 1. Frequencies (in rad/s) of the lowest-frequency hydroelastic vibration modes.

\begin{tabular}{cccccrcrc}
\hline Mode & \multicolumn{1}{c}{$N=6$} & \multicolumn{1}{c}{$N=7$} & \multicolumn{1}{c}{$N=8$} & \multicolumn{1}{c}{$N=9$} & $N=10$ & Rate & Extrapol. & Extrapol. $[6]$ \\
\hline 1 & 729.087 & 715.485 & 706.136 & 699.399 & 694.359 & 1.60 & 666.995 & 666.401 \\
2 & 2397.609 & 2372.312 & 2355.008 & 2342.639 & 2333.479 & 1.66 & 2285.324 & 2285.811 \\
3 & 4039.499 & 3991.105 & 3957.060 & 3932.129 & 3913.261 & 1.47 & 3799.831 & 3799.823 \\
4 & 4568.021 & 4556.431 & 4548.289 & 4542.284 & 4537.689 & 1.43 & 4509.573 & 4509.499 \\
5 & 5470.491 & 5444.295 & 5426.390 & 5413.570 & 5404.047 & 1.65 & 5353.859 & 5353.978 \\
\hline
\end{tabular}

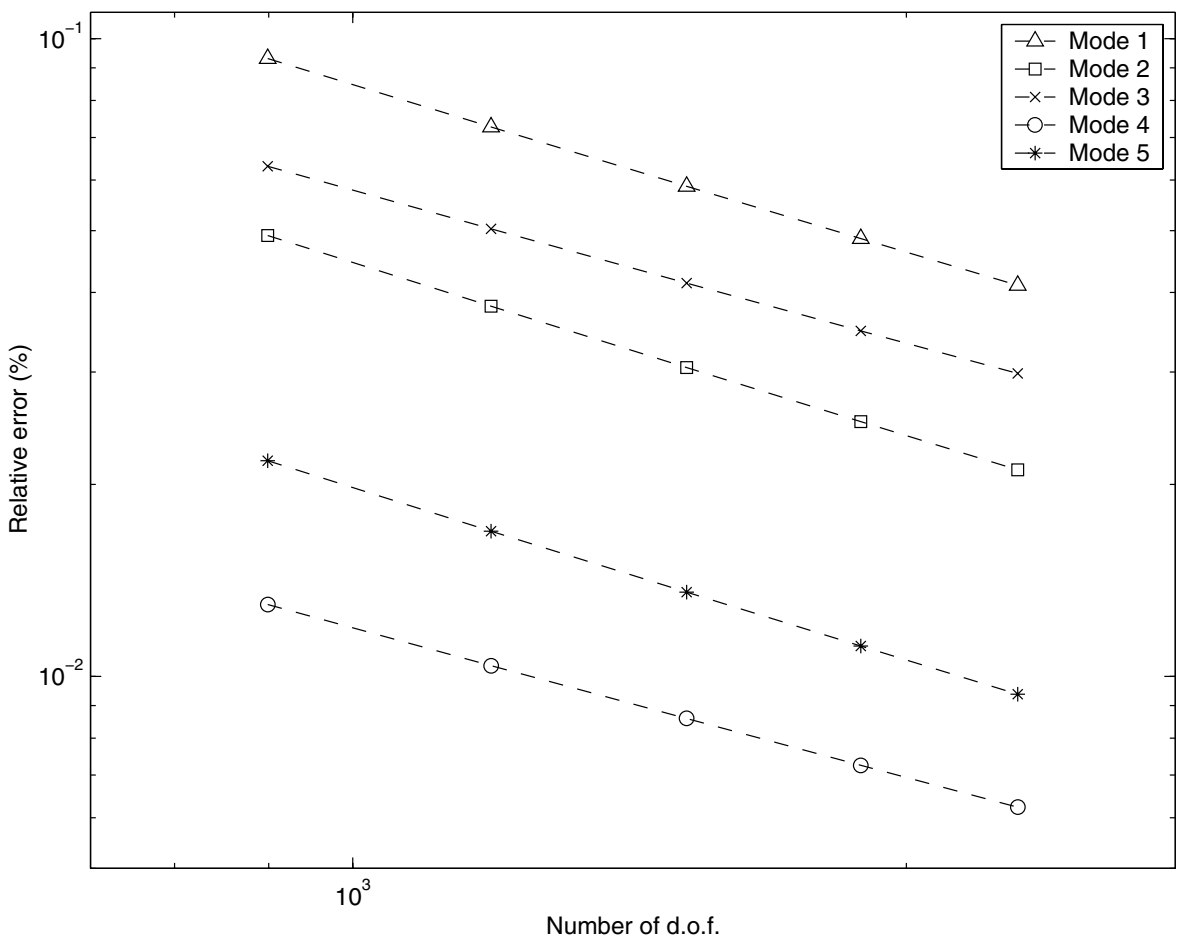

FIGURE 4. Error curves of the computed frequencies: log-log plot of relative error versus d.o.f.

The computed convergence rates are slightly better than those predicted by the theory. Indeed, the fluid domain is convex in this experiment, and hence $s=1$. On the other hand, the value of $t$ is determined by the strongest singularity of the elasticity equations on the domain $\Omega_{\mathrm{S}}$. In this case this happens at the external Dirichlet-Neumann $\frac{\pi}{2}$ corners which, for $\nu_{\mathrm{S}}=0.35$, is $t \approx 0.68$ (see [11]). Thus, according to Theorem 3.7 the order of convergence should be about 1.36. The reason why the experimental convergence rates are slightly better is that the computed vibration frequencies are yet in a preasymptotic range (see [1] where a similar experimental behavior is reported in the case of compressible fluids).

Figure 4 shows the error curve of each vibration mode; namely, a log-log plot of the relative error of the computed frequencies versus the number of degrees of freedom (d.o.f.) of the used mesh. The extrapolated frequencies have been used as "exact" to compute these errors.

Finally, Figures 5 to 9 show the solid deformation amplitudes obtained with the coarsest meshes for each vibration mode. 


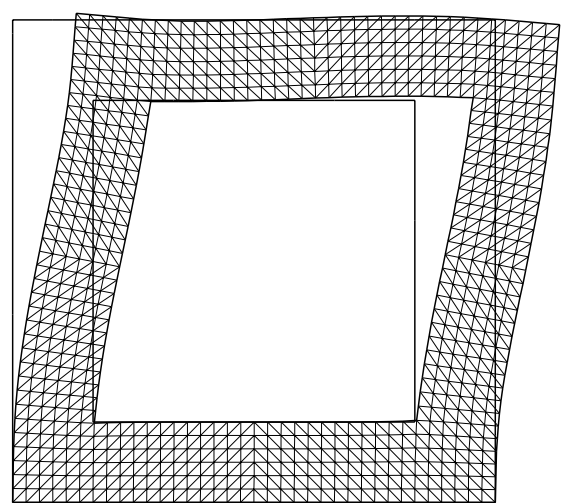

Figure 5. Solid deformation amplitude. First vibration mode. Mesh: $N=6$.

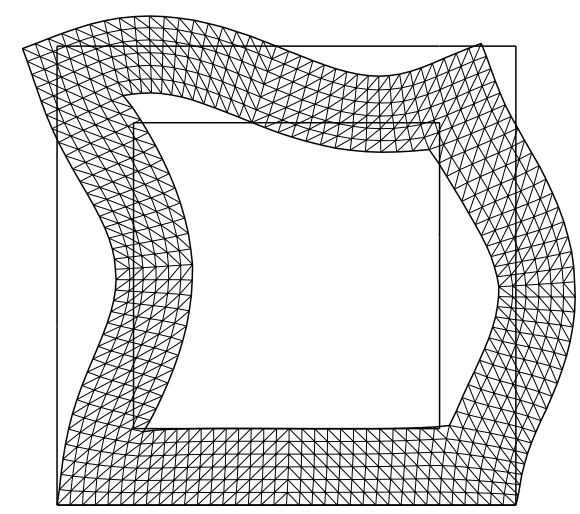

FiguRE 7. Solid deformation amplitude. Third vibration mode. Mesh: $N=6$.

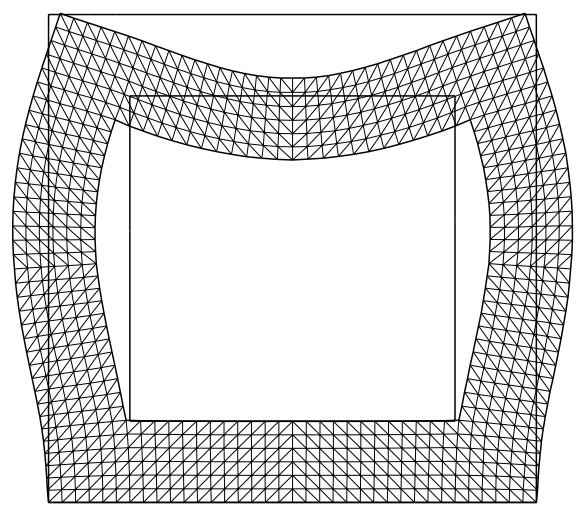

Figure 6. Solid deformation amplitude. Second vibration mode. Mesh: $N=6$.

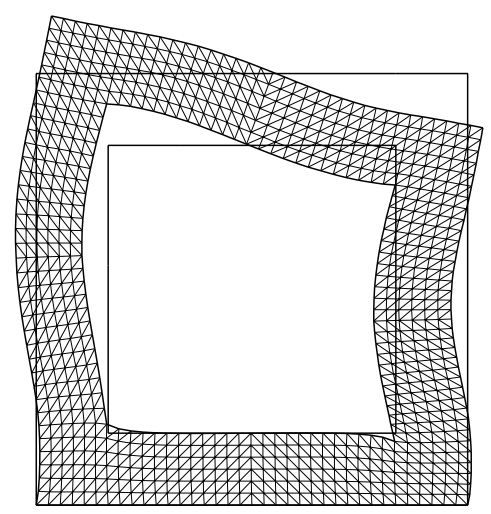

Figure 8. Solid deformation amplitude. Fourth vibration mode. Mesh: $N=6$.

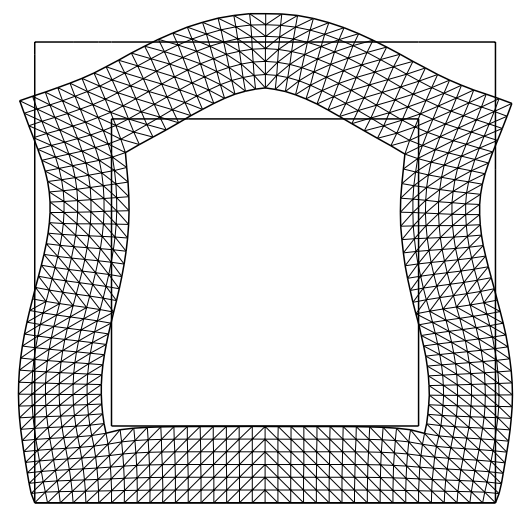

Figure 9. Solid deformation amplitude. Fifth vibration mode. Mesh: $N=6$. 
Acknowledgements. The authors thank Norbert Heuer for providing the software to compute the terms involving the boundary integral operators.

\section{REFERENCES}

[1] A. Alonso, A. Dello Russo, C. Otero-Souto, C. Padra and R. Rodríguez, An adaptive finite element scheme to solve fluidstructure vibration problems on non-matching grids. Comput. Visual. Sci. 4 (2001) 67-78.

[2] I. Babuška and J. Osborn, Eigenvalue problems, in Handbook of Numerical Analysis, P.G. Ciarlet and J.L. Lions Eds., Vol. II, North-Holland, Amsterdam (1991) 641-787.

[3] A. Bermúdez, R. Durán and R. Rodríguez, Finite element solution of incompressible fluid-structure vibration problems. Internat. J. Numer. Methods Eng. 40 (1997) 1435-1448.

[4] A. Bermúdez, R. Durán and R. Rodríguez, Finite element analysis of compressible and incompressible fluid-solid systems, Math. Comp. 67 (1998) 111-136.

[5] A. Bermúdez and R. Rodríguez, Finite element analysis of sloshing and hydroelastic vibrations under gravity. ESAIM: M2AN 33 (1999) 305-327.

[6] A. Bermúdez, R. Rodríguez and D. Santamarina, A finite element solution of an added mass formulation for coupled fluid-solid vibrations. Numer. Math. 87 (2000) 201-227.

[7] P.G. Ciarlet, Basic error estimates for elliptic problems, in Handbook of Numerical Analysis, P.G. Ciarlet and J.L. Lions Eds., Vol. II, North-Holland, Amsterdam (1991) 17-351.

[8] M. Costabel, Boundary integral operators on Lipschitz domains: Elementary results. SIAM J. Math. Anal. 19 (1988) 613-621.

[9] V.J. Ervin, N. Heuer and E.P. Stephan, On the $h-p$ version of the boundary element method for Symm's integral equation on polygons. Comput. Methods Appl. Mech. Eng. 110 (1993) 25-38.

[10] G.N. Gatica and G.C. Hsiao, Boundary-Field Equation Methods for a Class of Nonlinear Problems. Longman, Harlow, Pitman Res. Notes Math. Ser. 331 (1995).

[11] P. Grisvard, Elliptic Problems in Nonsmooth Domains. Pitman, Boston, MA, Monogr. Stud. Math. 24 (1985).

[12] M. Hamdi, Y. Ousset and G. Verchery, A displacement method for the analysis of vibrations of coupled fluid-structure systems. Internat. J. Numer. Methods Eng. 13 (1978) 139-150.

[13] G.C. Hsiao, On the boundary-field equation methods for fluid-structure interactions, in Problems and Methods in Mathematical Physics (Chemnitz, 1993), L. Jentsch and F. Tröltzsch, Eds. Teubner, Stuttgart, Teubner-Texte Math. 134 (1994) 79-88.

[14] G.C. Hsiao and W.L. Wendland, A finite element method for some integral equations of the first kind. J. Math. Anal. Appl. 58 (1977) 449-481.

[15] G.C. Hsiao, R.E. Kleinman and G.F. Roach, Weak solutions of fluid-solid interaction problems. Math. Nachr. 218 (2000) 139-163.

[16] G.C. Hsiao, R.E. Kleinman and L.S. Schuetz, On variational formulations of boundary value problems for fluid-solid interactions. Elastic Wave Propagation (Galway, 1988). North-Holland, Amsterdam, North-Holland Ser. Appl. Math. Mech. 35 (1989) 321-326.

[17] W. McLean, Strongly Elliptic Systems and Boundary Integral Equations. Cambridge University Press, Cambridge (2000).

[18] D. Mercier, Some systems of PDE on polygonal networks, in Partial Differential Equations on Multistructures (Luminy, 1999), F.A. Mehmeti, J. von Below and S. Nicaise Eds., Dekker, New York, Lect. Notes Pure Appl. Math. 219 (2001) 163-182.

[19] D. Mercier, Problèmes de transmission sur des réseaux polygonaux pour des systèmes d'EDP. Ann. Fac. Sci. Toulouse Math. 10 (2001) 107-162.

[20] H.J.-P. Morand and R. Ohayon, Fluid-Structure Interaction. J. Wiley \& Sons, Chichester (1995).

[21] P. Ryan, Eigenvalue and eigenfunction error estimates for finite element formulations of linear hydroelasticity. Math. Comp. 70 (2001) 471-487.

[22] M.E. Torrejón, Solución Numérica de Problemas de Vibraciones Hidroelásticas. Degree Thesis in Mathematical Engineering, Universidad de Concepción, Chile (2003).

[23] O.C. Zienkiewicz and R.L. Taylor, The Finite Element Method. Mc Graw Hill, London (1989). 